\title{
Superintegrability on Three-Dimensional Riemannian and Relativistic Spaces of Constant Curvature
}

\author{
Francisco José HERRANZ ${ }^{\dagger}$ and Ángel BALLESTEROS ${ }^{\ddagger}$ \\ † Departamento de Física, Escuela Politécnica Superior, Universidad de Burgos, \\ 09001 Burgos, Spain \\ E-mail: fjherranz@ubu.es \\ $¥$ Departamento de Física, Facultad de Ciencias, Universidad de Burgos, \\ 09001 Burgos, Spain \\ E-mail: angelb@ubu.es
}

Received December 21, 2005, in final form January 20, 2006; Published online January 24, 2006

Original article is available at http://www.emis.de/journals/SIGMA/2006/Paper010/

\begin{abstract}
A family of classical superintegrable Hamiltonians, depending on an arbitrary radial function, which are defined on the $3 \mathrm{D}$ spherical, Euclidean and hyperbolic spaces as well as on the (2+1)D anti-de Sitter, Minkowskian and de Sitter spacetimes is constructed. Such systems admit three integrals of the motion (besides the Hamiltonian) which are explicitly given in terms of ambient and geodesic polar coordinates. The resulting expressions cover the six spaces in a unified way as these are parametrized by two contraction parameters that govern the curvature and the signature of the metric on each space. Next two maximally superintegrable Hamiltonians are identified within the initial superintegrable family by finding the remaining constant of the motion. The former potential is the superposition of a (curved) central harmonic oscillator with other three oscillators or centrifugal barriers (depending on each specific space), so that this generalizes the Smorodinsky-Winternitz system. The latter one is a superposition of the Kepler-Coulomb potential with another two oscillators or centrifugal barriers. As a byproduct, the Laplace-Runge-Lenz vector for these spaces is deduced. Furthermore both potentials are analysed in detail for each particular space. Some comments on their generalization to arbitrary dimension are also presented.
\end{abstract}

Key words: integrable systems; curvature; contraction; harmonic oscillator; Kepler-Coulomb; hyperbolic; de Sitter

2000 Mathematics Subject Classification: 37J35; 22E60; 37J15; 70H06

\section{Introduction}

In [14] Evans obtained a classification of classical superintegrable systems [38] on the threedimensional (3D) Euclidean space $\mathbf{E}^{3}$. At this dimension he called minimally superintegrable systems those endowed with three constants of the motion besides the Hamiltonian, that is, they have one constant more than those necessary to ensure complete integrability, but one less than the necessary number to determine maximal superintegrability. Amongst the resulting potentials let us consider

$$
\mathcal{U}=\mathcal{F}(r)+\frac{\beta_{1}}{x^{2}}+\frac{\beta_{2}}{y^{2}}+\frac{\beta_{3}}{z^{2}}
$$

where $\mathcal{F}(r)$ is an arbitrary smooth function, the three $\beta_{i}$ are arbitrary real parameters, $(x, y, z)$ are Cartesian coordinates on $\mathbf{E}^{3}$, and $r=\sqrt{x^{2}+y^{2}+z^{2}}$. Thus this potential is formed by a central term with three centrifugal barriers. Next by analysing the radial function $\mathcal{F}(r)$ two 
relevant and well known expressions arise conveying the additional constant of the motion. These two cases then appear in the classification by Evans as maximally superintegrable systems as they have the maximum number of functionally independent constants of the motion, four ones plus the Hamiltonian. Explicitly, these are:

- The Smorodinsky-Winternitz $(\mathrm{SW})$ potential [17] when $\mathcal{F}(r)=\beta_{0} r^{2}$ :

$$
\mathcal{U}^{\mathrm{SW}}=\beta_{0}\left(x^{2}+y^{2}+z^{2}\right)+\frac{\beta_{1}}{x^{2}}+\frac{\beta_{2}}{y^{2}}+\frac{\beta_{3}}{z^{2}},
$$

which corresponds to the superposition of a harmonic oscillator with angular frequency $\sqrt{\beta_{0}}$ and the three centrifugal terms.

- And a generalized Kepler-Coulomb $(\mathrm{GKC})$ potential when $\mathcal{F}(r)=-k / r$ :

$$
\mathcal{U}^{\mathrm{GKC}}=-\frac{k}{\sqrt{x^{2}+y^{2}+z^{2}}}+\frac{\beta_{1}}{x^{2}}+\frac{\beta_{2}}{y^{2}},
$$

which is formed by the proper Kepler-Coulomb (KC) potential with parameter $k$ together with two of the famous centrifugal terms.

Superintegrable systems on $\mathbf{E}^{2}$ and $\mathbf{E}^{3}[14,35]$ have also been implemented on the two classical Riemannian spaces of constant curvature. In particular, some superintegrable systems on the 2D and 3D spheres, $\mathbf{S}^{2}$ and $\mathbf{S}^{3}$, have been studied in [20], on the hyperbolic plane $\mathbf{H}^{2}$ in [29, 30], while on $\mathbf{H}^{3}$ can be found in [21]. Moreover classifications of superintegrable systems on $\mathbf{S}^{2}$ and $\mathbf{H}^{2}$ have been carried out in $[28,31,34,39]$. These results contain the corresponding (curved) harmonic oscillator [26, 36] and $\mathrm{KC}$ potential [43], which in arbitrary dimension correspond, in this order, to the following radial potential

$$
\mathcal{F}(r)=\left\{\begin{array}{lll}
\beta_{0} \tan ^{2} r, & \text { on } & \mathbf{S}^{N} ; \\
\beta_{0} r^{2}, & \text { on } & \mathbf{E}^{N} ; \\
\beta_{0} \tanh ^{2} r, & \text { on } & \mathbf{H}^{N}
\end{array} \quad \mathcal{F}(r)=\left\{\begin{array}{lll}
-k / \tan r, & \text { on } & \mathbf{S}^{N} \\
-k / r, & \text { on } & \mathbf{E}^{N} \\
-k / \tanh r, & \text { on } & \mathbf{H}^{N}
\end{array}\right.\right.
$$

We recall that the SW system on $\mathbf{S}^{N}$ and $\mathbf{H}^{N}$ have been constructed in $[9,23]$ (curved harmonic oscillator plus $N$ terms) showing that this keeps maximal superintegrability for any value of the curvature.

However, as far as we know, the construction of the GKC potential on $\mathbf{S}^{N}$ and $\mathbf{H}^{N}$ as well as which are the corresponding SW and GKC systems on the relativistic spacetimes of constant curvature is still lacking, that is, also covering the anti-de Sitter, Minkowskian and de Sitter spacetimes. The aim of this paper is to present all of these Hamiltonians on these six 3D spaces in a unified setting by making use of two explicit contraction parameters which determine the curvature and the signature of the metric. In this sense, the results here presented can be considered as the cornerstone for a further generalization of all of these systems to arbitrary dimension. In this respect, we would like to mention that although very recently such potentials have been deduced on the $(1+1) \mathrm{D}$ relativistic spacetimes $[8,12]$, this low dimension does not show the guide for a direct generalization to $N D$.

The structure of this paper is as follows. The next section contains the necessary basics on the Lie groups of isometries on the six spaces together with the two coordinate systems we shall deal with throughout the paper: ambient (Weierstrass) coordinates in an auxiliary linear space $\mathbb{R}^{4}$ and intrinsic geodesic polar (spherical) coordinates. The kinetic energy determining the geodesic motion is then studied in Section 3 by starting from the metric. The generalization of the Euclidean family (1.1) to these spaces is developed in Section 4 in such a manner that general and global expressions for the Hamiltonian and its three integrals of motion are explicitly given. 
The next two sections are devoted to the study of two maximal superintegrable Hamiltonians arising in the above family by choosing in an adequate way the radial function $\mathcal{F}(r)$ (fulfilling (1.4) for the Riemannian spaces) and finding at the same time the remaining constant of the motion. In this way we obtain the generalization of the SW (1.2) and GKC (1.3) potentials for any value of the curvature and signature of the metric. Furthermore a detail description of such systems is performed on each particular space. We stress that, by following the geometrical interpretation formerly introduced in $[40,41,42]$ and generalized in $[8,9,23]$, the SW potential is interpreted as the superposition of a central harmonic oscillator with three non-central oscillators or centrifugal barriers according to each specific space. Likewise, the GKC system can be seen as the superposition of the $\mathrm{KC}$ potential with two oscillators or centrifugal barriers; in this case, we moreover deduce the corresponding Laplace-Runge-Lenz vector. Finally, some remarks and comments mainly concerning the pattern for the construction of such systems for arbitrary dimension close the paper.

\section{Riemannian spaces and relativistic spacetimes}

Let us consider a subset of real Lie algebras contained in the family of the Cayley-Klein orthogonal algebras $[4,18]$. These can also be obtained as the $\mathbb{Z}_{2} \otimes \mathbb{Z}_{2}$ graded contractions of so(4) and are denoted $s o_{\kappa_{1}, \kappa_{2}}(4)$ where $\kappa_{1}$ and $\kappa_{2}$ are two real contraction parameters. The Lie brackets of $s o_{\kappa_{1}, \kappa_{2}}(4)$ in the basis spanned by $\left\{J_{\mu \nu}\right\}$ where $\mu, \nu=0,1,2,3$ and $\mu<\nu$ read [4]

$$
\begin{aligned}
& {\left[J_{12}, J_{13}\right]=\kappa_{2} J_{23}, \quad\left[J_{12}, J_{23}\right]=-J_{13}, \quad\left[J_{13}, J_{23}\right]=J_{12},} \\
& {\left[J_{12}, J_{01}\right]=J_{02}, \quad\left[J_{13}, J_{01}\right]=J_{03}, \quad\left[J_{23}, J_{02}\right]=J_{03} \text {, }} \\
& {\left[J_{12}, J_{02}\right]=-\kappa_{2} J_{01}, \quad\left[J_{13}, J_{03}\right]=-\kappa_{2} J_{01}, \quad\left[J_{23}, J_{03}\right]=-J_{02} \text {, }} \\
& {\left[J_{01}, J_{02}\right]=\kappa_{1} J_{12}, \quad\left[J_{01}, J_{03}\right]=\kappa_{1} J_{13}, \quad\left[J_{02}, J_{03}\right]=\kappa_{1} \kappa_{2} J_{23} \text {, }} \\
& {\left[J_{01}, J_{23}\right]=0, \quad\left[J_{02}, J_{13}\right]=0, \quad\left[J_{03}, J_{12}\right]=0 .}
\end{aligned}
$$

There are two Casimir invariants

$$
\begin{aligned}
& \mathcal{C}_{1}=\kappa_{2} J_{01}^{2}+J_{02}^{2}+J_{03}^{2}+\kappa_{1} J_{12}^{2}+\kappa_{1} J_{13}^{2}+\kappa_{1} \kappa_{2} J_{23}^{2}, \\
& \mathcal{C}_{2}=\kappa_{2} J_{01} J_{23}-J_{02} J_{13}+J_{03} J_{12},
\end{aligned}
$$

where $\mathcal{C}_{1}$ is associated to the Killing-Cartan form.

Let us explain the geometrical role of the contraction parameters $\kappa_{1}$ and $\kappa_{2}$. The involutive automorphisms defined by

$$
\begin{aligned}
& \Theta_{0}: \quad J_{i j} \rightarrow J_{i j}, \quad J_{0 i} \rightarrow-J_{0 i}, \quad i=1,2,3, \\
& \Theta_{01}:\left\{J_{01}, J_{23}\right\} \rightarrow\left\{J_{01}, J_{23}\right\}, \quad\left\{J_{0 j}, J_{1 j}\right\} \rightarrow-\left\{J_{0 j}, J_{1 j}\right\}, \quad j=2,3,
\end{aligned}
$$

generate a $\mathbb{Z}_{2} \otimes \mathbb{Z}_{2}$-grading of $s o_{\kappa_{1}, \kappa_{2}}(4)$ in such a manner that $\kappa_{1}$ and $\kappa_{2}$ are two graded contraction parameters coming from the $\mathbb{Z}_{2}$-grading determined by $\Theta_{0}$ and $\Theta_{01}$, respectively. By scaling the Lie generators each parameter $\kappa_{i}$ can be reduced to either $+1,0$ or -1 ; the vanishment of $\kappa_{i}$ is equivalent to apply an Inönü-Wigner contraction.

Furthermore, these automorphisms give rise to the following Cartan decompositions:

$$
\begin{array}{lll}
s o_{\kappa_{1}, \kappa_{2}}(4)=\mathfrak{h}_{0} \oplus \mathfrak{p}_{0}, & \mathfrak{h}_{0}=\left\langle J_{12}, J_{13}, J_{23}\right\rangle=s o_{\kappa_{2}}(3), & \mathfrak{p}_{0}=\left\langle J_{01}, J_{02}, J_{03}\right\rangle, \\
s o_{\kappa_{1}, \kappa_{2}}(4)=\mathfrak{h}_{01} \oplus \mathfrak{p}_{01}, & \mathfrak{h}_{01}=\left\langle J_{01}, J_{23}\right\rangle=s o_{\kappa_{1}}(2) \oplus s o(2), & \mathfrak{p}_{01}=\left\langle J_{02}, J_{03}, J_{12}, J_{13}\right\rangle
\end{array}
$$

If $H_{0}$ and $H_{01}$ denote the Lie subgroups with Lie algebras $\mathfrak{h}_{0}$ and $\mathfrak{h}_{01}$, we obtain two families of symmetric homogeneous spaces [22], namely the usual 3D space of points $S O_{\kappa_{1}, \kappa_{2}}(4) / H_{0}$ 
and the $4 \mathrm{D}$ space of lines $S O_{\kappa_{1}, \kappa_{2}}(4) / H_{01}$, which have constant curvature equal to $\kappa_{1}$ and $\kappa_{2}$, respectively.

We shall make use of the former space which has a metric with a signature governed by $\kappa_{2}$ as $\operatorname{diag}\left(+1, \kappa_{2}, \kappa_{2}\right)$ and we denote it

$$
\mathbb{S}_{\left[\kappa_{1}\right] \kappa_{2}}^{3}=S O_{\kappa_{1}, \kappa_{2}}(4) / S O_{\kappa_{2}}(3) .
$$

Thus when $\kappa_{2}$ is positive we recover the three classical Riemannian spaces, while if this is negative we find a Lorentzian metric. In this case, there is a kinematical interpretation for the homogeneous spaces. Let $P_{0}, P_{i}, K_{i}$ and $J(i=1,2)$ the usual generators of time translation, space translations, boosts and spatial rotations, respectively. Under the following identification

$$
P_{0}=J_{01}, \quad P_{i}=J_{0 i+1}, \quad K_{i}=J_{1 i+1}, \quad J=J_{23}, \quad i=1,2,
$$

the three algebras with $\kappa_{2}=-1 / c^{2}<0$ ( $c$ is the speed of light) are the Lie algebras of the groups of motions of $(2+1) \mathrm{D}$ relativistic spacetime models. Thus the commutation relations (2.1) read now

$$
\begin{array}{llll}
{\left[J, K_{i}\right]=\epsilon_{i j} K_{j},} & {\left[K_{1}, K_{2}\right]=-\frac{1}{c^{2}} J,} & {\left[P_{0}, K_{i}\right]=-P_{i},} & {\left[P_{i}, K_{j}\right]=-\frac{1}{c^{2}} \delta_{i j} P_{0},} \\
{\left[J, P_{i}\right]=\epsilon_{i j} P_{j},} & {\left[P_{1}, P_{2}\right]=-\frac{\kappa_{1}}{c^{2}} J,} & {\left[P_{0}, P_{i}\right]=\kappa_{1} K_{i},} & {\left[P_{0}, J_{i}\right]=0,}
\end{array}
$$

where $\epsilon_{i j}$ is a skew-symmetric tensor such that $\epsilon_{12}=1$. In this framework the curvature of the spacetime can be written in terms of the (time) universe radius $\tau$ as $\kappa_{1}= \pm 1 / \tau^{2}$ (which is also proportional to the cosmological constant). The Casimir invariants $(2.2), \mathcal{C}_{1}$ and $\mathcal{C}_{2}$, correspond to the energy and angular momentum of a particle in the free kinematics of the relativistic spacetime:

$$
\begin{aligned}
& \mathcal{C}_{1}=-\frac{1}{c^{2}} P_{0}^{2}+P_{1}^{2}+P_{2}^{2}+\kappa_{1}\left(K_{1}^{2}+K_{2}^{2}\right)-\frac{\kappa_{1}}{c^{2}} J^{2}, \\
& \mathcal{C}_{2}=-\frac{1}{c^{2}} P_{0} J-P_{1} K_{2}+P_{2} K_{1} .
\end{aligned}
$$

On the other hand, if $\kappa_{2}=0$ we obtain a degenerate metric which corresponds to Newtonian spacetimes. Since our aim is to construct superintegrable systems on these homogeneous spaces, for which the kinetic energy is provided by the metric, we avoid the contraction $\kappa_{2}=0$. The resulting six particular spaces contained in the family $\mathbb{S}_{\left[\kappa_{1}\right] \kappa_{2}}^{3}$ are displayed in Table 1.

Table 1. $3 \mathrm{D}$ symmetric homogeneous spaces $\mathbb{S}_{\left[\kappa_{1}\right] \kappa_{2}}^{3}=S O_{\kappa_{1}, \kappa_{2}}(4) / S O_{\kappa_{2}}(3)$ and their metric in geodesic polar coordinates according to $\kappa_{1} \in\{+1,0,-1\}$ and $\kappa_{2} \in\{+1,-1\}$.

\begin{tabular}{ll}
\hline 3 D Riemannian spaces & $(2+1)$ D Relativistic spacetimes \\
\hline$\bullet$ Spherical space $\mathbf{S}^{3}$ & $\bullet$ Anti-de Sitter spacetime AdS $\mathbf{A}^{2+1}$ \\
$\mathbb{S}_{[+]+}^{3}=S O(4) / S O(3)$ & $\mathbb{S}_{[+]-}^{3}=S O(2,2) / S O(2,1)$ \\
$\mathrm{d} s^{2}=\mathrm{d} r^{2}+\sin ^{2} r \mathrm{~d} \theta^{2}+\sin ^{2} r \sin ^{2} \theta \mathrm{d} \phi^{2}$ & $\mathrm{~d} s^{2}=\mathrm{d} r^{2}-\sin ^{2} r \mathrm{~d} \theta^{2}-\sin ^{2} r \sinh ^{2} \theta \mathrm{d} \phi^{2}$ \\
$\bullet$ Euclidean space $\mathbf{E}^{3}$ & $\bullet$ Minkowskian spacetime $\mathbf{M}^{2+1}$ \\
$\mathbb{S}_{[0]+}^{3}=I S O(3) / S O(3)$ & $\mathbb{S}_{[0]-}^{3}=I S O(2,1) / S O(2,1)$ \\
$\mathrm{d} s^{2}=\mathrm{d} r^{2}+r^{2} \mathrm{~d} \theta^{2}+r^{2} \sin ^{2} \theta \mathrm{d} \phi^{2}$ & $\mathrm{~d} s^{2}=\mathrm{d} r^{2}-r^{2} \mathrm{~d} \theta^{2}-r^{2} \sinh ^{2} \theta \mathrm{d} \phi^{2}$ \\
$\bullet$ Hyperbolic space $\mathbf{H}^{3}$ & $\bullet$ De Sitter spacetime $\mathbf{d} \mathbf{S}^{2+1}$ \\
$\mathbb{S}_{[-]+}^{3}=S O(3,1) / S O(3)$ & $\mathbb{S}_{[-]-}^{3}=S O(3,1) / S O(2,1)$ \\
$\mathrm{d} s^{2}=\mathrm{d} r^{2}+\sinh ^{2} r \mathrm{~d} \theta^{2}+\sinh ^{2} r \sin ^{2} \theta \mathrm{d} \phi^{2}$ & $\mathrm{~d} s^{2}=\mathrm{d} r^{2}-\sinh ^{2} r \mathrm{~d} \theta^{2}-\sinh ^{2} r \sinh ^{2} \theta \mathrm{d} \phi^{2}$ \\
\hline
\end{tabular}




\subsection{Vector model and ambient coordinates}

The vector representation of $s o_{\kappa_{1}, \kappa_{2}}(4)$ is given by the following $4 \times 4$ real matrices [4]:

$$
\begin{aligned}
& J_{01}=\left(\begin{array}{cccc}
\cdot & -\kappa_{1} & \cdot & \cdot \\
1 & \cdot & \cdot & \cdot \\
\cdot & \cdot & \cdot & \cdot \\
\cdot & \cdot & \cdot & \cdot
\end{array}\right), \quad J_{12}=\left(\begin{array}{cccc}
\cdot & \cdot & \cdot & \cdot \\
\cdot & \cdot & -\kappa_{2} & \cdot \\
\cdot & 1 & \cdot & \cdot \\
\cdot & \cdot & \cdot & \cdot
\end{array}\right), \\
& J_{02}=\left(\begin{array}{cccc}
\cdot & \cdot & -\kappa_{1} \kappa_{2} & \cdot \\
\cdot & \cdot & \cdot & \cdot \\
1 & \cdot & \cdot & \cdot \\
\cdot & \cdot & \cdot & \cdot
\end{array}\right), \quad J_{13}=\left(\begin{array}{cccc}
\cdot & \cdot & \cdot & \cdot \\
\cdot & \cdot & \cdot & -\kappa_{2} \\
\cdot & \cdot & \cdot & \cdot \\
\cdot & 1 & \cdot & \cdot
\end{array}\right) \text {, }
\end{aligned}
$$

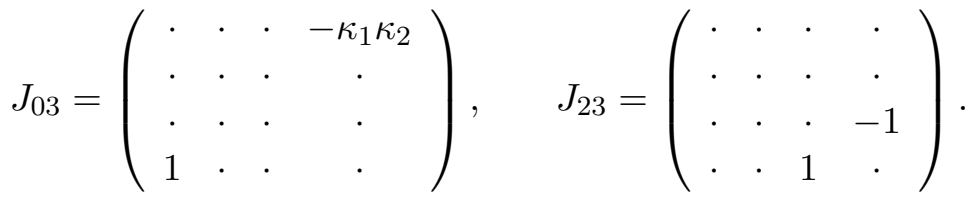

Their exponential provides the corresponding one-parametric subgroups of $S O_{\kappa_{1}, \kappa_{2}}(4)$ :

$$
\begin{aligned}
& \mathrm{e}^{x J_{01}}=\left(\begin{array}{cccc}
\mathrm{C}_{\kappa_{1}}(x) & -\kappa_{1} \mathrm{~S}_{\kappa_{1}}(x) & \cdot & \cdot \\
\mathrm{S}_{\kappa_{1}}(x) & \mathrm{C}_{\kappa_{1}}(x) & \cdot & \cdot \\
\cdot & \cdot & 1 & \cdot \\
\cdot & \cdot & \cdot & 1
\end{array}\right), \quad \mathrm{e}^{x J_{12}}=\left(\begin{array}{cccc}
1 & \cdot & \cdot & \cdot \\
\cdot & \mathrm{C}_{\kappa_{2}}(x) & -\kappa_{2} \mathrm{~S}_{\kappa_{2}}(x) & \cdot \\
\cdot & \mathrm{S}_{\kappa_{2}}(x) & \mathrm{C}_{\kappa_{2}}(x) & \cdot \\
\cdot & \cdot & \cdot & 1
\end{array}\right), \\
& \mathrm{e}^{x J_{02}}=\left(\begin{array}{cccc}
\mathrm{C}_{\kappa_{1} \kappa_{2}}(x) & \cdot & -\kappa_{1} \kappa_{2} \mathrm{~S}_{\kappa_{1} \kappa_{2}}(x) & \cdot \\
\cdot & 1 & \cdot & \cdot \\
\mathrm{S}_{\kappa_{1} \kappa_{2}}(x) & \cdot & \mathrm{C}_{\kappa_{1} \kappa_{2}}(x) & \cdot \\
\cdot & \cdot & \cdot & 1
\end{array}\right), \quad \mathrm{e}^{x J_{13}}=\left(\begin{array}{cccc}
1 & \cdot & \cdot & \cdot \\
\cdot & \mathrm{C}_{\kappa_{2}}(x) & \cdot & -\kappa_{2} \mathrm{~S}_{\kappa_{2}}(x) \\
\cdot & \cdot & 1 & \cdot \\
\cdot & \mathrm{S}_{\kappa_{2}}(x) & \cdot & \mathrm{C}_{\kappa_{2}}(x)
\end{array}\right) \text {, } \\
& \mathrm{e}^{x J_{03}}=\left(\begin{array}{cccc}
\mathrm{C}_{\kappa_{1} \kappa_{2}}(x) & \cdot & \cdot & -\kappa_{1} \kappa_{2} \mathrm{~S}_{\kappa_{1} \kappa_{2}}(x) \\
\cdot & 1 & \cdot & \cdot \\
\cdot & \cdot & 1 & \cdot \\
\mathrm{S}_{\kappa_{1} \kappa_{2}}(x) & \cdot & \cdot & \mathrm{C}_{\kappa_{1} \kappa_{2}}(x)
\end{array}\right), \quad \mathrm{e}^{x J_{23}}=\left(\begin{array}{cccc}
1 & \cdot & \cdot & \cdot \\
\cdot & 1 & \cdot & \cdot \\
\cdot & \cdot & \cos x & -\sin x \\
\cdot & \cdot & \sin x & \cos x
\end{array}\right),
\end{aligned}
$$

where we have introduced the $\kappa$-dependent cosine and sine functions defined by $[3,5]$

$$
\begin{aligned}
& \mathrm{C}_{\kappa}(x)=\sum_{l=0}^{\infty}(-\kappa)^{l} \frac{x^{2 l}}{(2 l) !}=\left\{\begin{array}{cc}
\cos \sqrt{\kappa} x, & \kappa>0 ; \\
1, & \kappa=0 ; \\
\cosh \sqrt{-\kappa} x, & \kappa<0,
\end{array}\right. \\
& \mathrm{S}_{\kappa}(x)=\sum_{l=0}^{\infty}(-\kappa)^{l} \frac{x^{2 l+1}}{(2 l+1) !}=\left\{\begin{array}{cc}
\frac{1}{\sqrt{\kappa}} \sin \sqrt{\kappa} x, & \kappa>0 ; \\
x, & \kappa=0 ; \\
\frac{1}{\sqrt{-\kappa}} \sinh \sqrt{-\kappa} x, & \kappa<0 .
\end{array}\right.
\end{aligned}
$$

Notice that $\kappa \in\left\{\kappa_{1}, \kappa_{1} \kappa_{2}, \kappa_{2}\right\}$. The tangent is defined as $\mathrm{T}_{\kappa}(x)=\mathrm{S}_{\kappa}(x) / \mathrm{C}_{\kappa}(x)$. Properties and trigonometric relations for these $\kappa$-functions, which are necessary in the further computations, can be found in [24, 25]; for instance,

$$
\mathrm{C}_{\kappa}^{2}(x)+\kappa \mathrm{S}_{\kappa}^{2}(x)=1, \quad \frac{\mathrm{d}}{\mathrm{d} x} \mathrm{C}_{\kappa}(x)=-\kappa \mathrm{S}_{\kappa}(x), \quad \frac{\mathrm{d}}{\mathrm{d} x} \mathrm{~S}_{\kappa}(x)=\mathrm{C}_{\kappa}(x) .
$$

Under the above matrix algebra and group representations it is verified that

$$
X^{T} \mathbb{I}_{\kappa}+\mathbb{I}_{\kappa} X=0, \quad X \in s o_{\kappa_{1}, \kappa_{2}}(4), \quad Y^{T} \mathbb{I}_{\kappa} Y=\mathbb{I}_{\kappa}, \quad Y \in S O_{\kappa_{1}, \kappa_{2}}(4),
$$


( $X^{T}$ is the transpose matrix of $X$ ) with respect to the bilinear form

$$
\mathbb{I}_{\kappa}=\operatorname{diag}\left(+1, \kappa_{1}, \kappa_{1} \kappa_{2}, \kappa_{1} \kappa_{2}\right) .
$$

Therefore $S O_{\kappa_{1}, \kappa_{2}}(4)$ is a group of isometries of $\mathbb{I}_{\kappa}$ acting on a linear ambient space $\mathbb{R}^{4}=$ $\left(x_{0}, x_{1}, x_{2}, x_{3}\right)$ through matrix multiplication. The origin $O$ in $\mathbb{S}_{\left[\kappa_{1}\right] \kappa_{2}}^{3}$ has ambient coordinates $O=(1,0,0,0)$ and this point is invariant under the subgroup $H_{0}=S O_{\kappa_{2}}(3)=\left\langle J_{12}, J_{13}, J_{23}\right\rangle$ (see $(2.7))$. The orbit of $O$ corresponds to the homogeneous space $\mathbb{S}_{\left[\kappa_{1}\right] \kappa_{2}}^{3}$ which is contained in the "sphere"

$$
\Sigma \equiv x_{0}^{2}+\kappa_{1} x_{1}^{2}+\kappa_{1} \kappa_{2} x_{2}^{2}+\kappa_{1} \kappa_{2} x_{3}^{2}=1,
$$

determined by $\mathbb{I}_{\kappa}$. The ambient coordinates $\left(x_{0}, x_{1}, x_{2}, x_{3}\right)$, subjected to (2.8), are also called

Weierstrass coordinates. The metric on $\mathbb{S}_{\left[\kappa_{1}\right] \kappa_{2}}^{3}$ follows from the flat ambient metric in $\mathbb{R}^{4}$ divided by the curvature and restricted to $\Sigma$ :

$$
\mathrm{d} s^{2}=\left.\frac{1}{\kappa_{1}}\left(\mathrm{~d} x_{0}^{2}+\kappa_{1} \mathrm{~d} x_{1}^{2}+\kappa_{1} \kappa_{2} \mathrm{~d} x_{2}^{2}+\kappa_{1} \kappa_{2} \mathrm{~d} x_{3}^{2}\right)\right|_{\Sigma} .
$$

A differential realization of $s o_{\kappa_{1}, \kappa_{2}}(4)$, fulfilling (2.1), as first-order vector fields in the ambient coordinates is provided by the vector representation (2.6) and reads

$$
\begin{array}{ll}
J_{01}=\kappa_{1} x_{1} \partial_{0}-x_{0} \partial_{1}, & J_{0 j}=\kappa_{1} \kappa_{2} x_{j} \partial_{0}-x_{0} \partial_{j}, \\
J_{23}=x_{3} \partial_{2}-x_{2} \partial_{3}, & J_{1 j}=\kappa_{2} x_{j} \partial_{1}-x_{1} \partial_{j},
\end{array}
$$

where $j=2,3$ and $\partial_{\mu}=\partial / \partial x_{\mu}$.

\subsection{Geodesic polar coordinate system}

Let us consider a point $Q$ in $\mathbb{S}_{\left[\kappa_{1}\right] \kappa_{2}}^{3}$ with Weierstrass coordinates $\left(x_{0}, x_{1}, x_{2}, x_{3}\right)$. This can be parametrized in terms of three intrinsic quantities of the space itself in different ways. We shall make use of the geodesic polar coordinates $(r, \theta, \phi)$ which are defined through the following action of the one-parametric subgroups (2.7) on the origin $O=(1,0,0,0)$ :

$$
\begin{aligned}
& Q(r, \theta, \phi)=\exp \left\{\phi J_{23}\right\} \exp \left\{\theta J_{12}\right\} \exp \left\{r J_{01}\right\} O, \\
& \left(\begin{array}{c}
x_{0} \\
x_{1} \\
x_{2} \\
x_{3}
\end{array}\right)=\left(\begin{array}{c}
\mathrm{C}_{\kappa_{1}}(r) \\
\mathrm{S}_{\kappa_{1}}(r) \mathrm{C}_{\kappa_{2}}(\theta) \\
\mathrm{S}_{\kappa_{1}}(r) \mathrm{S}_{\kappa_{2}}(\theta) \cos \phi \\
\mathrm{S}_{\kappa_{1}}(r) \mathrm{S}_{\kappa_{2}}(\theta) \sin \phi
\end{array}\right) .
\end{aligned}
$$

Let $l_{1}$ be a (time-like) geodesic and $l_{2}, l_{3}$ two other (space-like) geodesics in $\mathbb{S}_{\left[\kappa_{1}\right] \kappa_{2}}^{3}$ orthogonal at $O$ in such a manner that each translation generator $J_{0 i}$ moves the origin along $l_{i}$. Then the (physical) geometrical meaning of the coordinates $(r, \theta, \phi)$ is as follows.

- The radial coordinate $r$ is the distance between $Q$ and $O$ measured along the (time-like) geodesic $l$ that joins both points. In the curved Riemannian spaces with $\kappa_{1}= \pm 1 / R^{2}, r$ has dimensions of length, $[r]=[R]$; notice however that the dimensionless coordinate $r / R$ is usually taken instead of $r$, and so the former is considered as an ordinary angle (see, e.g., [27]). In the relativistic spacetimes with $\kappa_{1}= \pm 1 / \tau^{2}, r$ has dimensions of a time-like length, that is, $[r]=[\tau]$.

- The coordinate $\theta$ is an ordinary angle in the three Riemannian spaces $\left(\kappa_{2}=+1\right)$ and this parametrizes the orientation of $l$ with respect to $l_{1}$, whilst $\theta$ corresponds to a rapidity in the spacetimes $\left(\kappa_{2}=-1 / c^{2}\right)$ with dimensions $[\theta]=[c]$. 
- Finally, $\phi$ is an ordinary angle for the six spaces that determines the orientation of $l$ with respect to the reference flag spanned by $l_{1}$ and $l_{2}$, that is, the 2-plane $l_{1} l_{2}$.

In the Riemannian spaces $(r, \theta, \phi)$ parametrize the complete space, while in the spacetimes these only cover the time-like region (in ambient coordinates this is $x_{2}^{2}+x_{3}^{2} \leq x_{1}^{2}$ ) limited by the light-cone on which $\theta \rightarrow \infty$. The flat contraction $\kappa_{1}=0$ gives rise to the usual spherical coordinates in the Euclidean space $\left(\kappa_{2}=1\right)$.

By introducing the parametrization (2.11) in the metric written in terms of ambient coordinates $(2.9)$ we obtain that

$$
\mathrm{d} s^{2}=\mathrm{d} r^{2}+\kappa_{2} \mathrm{~S}_{\kappa_{1}}^{2}(r)\left(\mathrm{d} \theta^{2}+\mathrm{S}_{\kappa_{2}}^{2}(\theta) \mathrm{d} \phi^{2}\right),
$$

which is particularized in Table 1 to each space. From it we compute the Levi-Civita connection $\Gamma_{i j}^{k}$, the Riemann $R_{j k l}^{i}$ and Ricci $R_{i j}$ tensors [13]. Their nonzero components are given by

$$
\begin{aligned}
& \Gamma_{\theta r}^{\theta}=\Gamma_{\phi r}^{\phi}=1 / \mathrm{T}_{\kappa_{1}}(r), \quad \Gamma_{\phi \theta}^{\phi}=1 / \mathrm{T}_{\kappa_{2}}(\theta), \quad \Gamma_{\theta \theta}^{r}=-\kappa_{2} \mathrm{~S}_{\kappa_{1}}(r) \mathrm{C}_{\kappa_{1}}(r), \\
& \Gamma_{\phi \phi}^{r}=-\kappa_{2} \mathrm{~S}_{\kappa_{1}}(r) \mathrm{C}_{\kappa_{1}}(r) \mathrm{S}_{\kappa_{2}}^{2}(\theta), \quad \Gamma_{\phi \phi}^{\theta}=-\mathrm{S}_{\kappa_{2}}(\theta) \mathrm{C}_{\kappa_{2}}(\theta), \\
& R_{\theta r \theta}^{r}=R_{\theta \phi \theta}^{\phi}=\kappa_{1} \kappa_{2} \mathrm{~S}_{\kappa_{1}}^{2}(r), \quad R_{\phi r \phi}^{r}=R_{\phi \theta \phi}^{\theta}=\kappa_{1} \kappa_{2} \mathrm{~S}_{\kappa_{1}}^{2}(r) \mathrm{S}_{\kappa_{2}}^{2}(\theta), \quad R_{r \theta r}^{\theta}=R_{r \phi r}^{\phi}=\kappa_{1}, \\
& R_{r r}=2 \kappa_{1}, \quad R_{\theta \theta}=2 \kappa_{1} \kappa_{2} \mathrm{~S}_{\kappa_{1}}^{2}(r), \quad R_{\phi \phi}=2 \kappa_{1} \kappa_{2} \mathrm{~S}_{\kappa_{1}}^{2}(r) \mathrm{S}_{\kappa_{2}}^{2}(\theta) .
\end{aligned}
$$

Therefore all the sectional curvatures turn out to be constant $K_{i j}=\kappa_{1}$, while the scalar curvature reads $K=6 \kappa_{1}$.

\section{Geodesic motion}

The metric (2.12) can be read as the kinetic energy of a particle written in terms of the velocities $(\dot{r}, \dot{\theta}, \dot{\phi})$, that is, the Lagrangian of the geodesic motion on the space $\mathbb{S}_{\left[\kappa_{1}\right] \kappa_{2}}^{3}$ given by

$$
\mathcal{T}=\frac{1}{2}\left(\dot{r}^{2}+\kappa_{2} \mathrm{~S}_{\kappa_{1}}^{2}(r)\left(\dot{\theta}^{2}+\mathrm{S}_{\kappa_{2}}^{2}(\theta) \dot{\phi}^{2}\right)\right) \text {. }
$$

Then the canonical momenta $\left(p_{r}, p_{\theta}, p_{\phi}\right)$ are obtained through $p=\partial \mathcal{T} / \partial \dot{q}(\dot{q}=\dot{r}, \dot{\theta}, \dot{\phi})$, namely,

$$
\begin{aligned}
& p_{r}=\dot{r} \\
& p_{\theta}=\kappa_{2} \mathrm{~S}_{\kappa_{1}}^{2}(r) \dot{\theta}, \\
& p_{\phi}=\kappa_{2} \mathrm{~S}_{\kappa_{1}}^{2}(r) \mathrm{S}_{\kappa_{2}}^{2}(\theta) \dot{\phi},
\end{aligned}
$$

so that the free Hamiltonian in the geodesic polar phase space $(q ; p)=\left(r, \theta, \phi ; p_{r}, p_{\theta}, p_{\phi}\right)$ with respect to the canonical Lie-Poisson bracket,

$$
\{f, g\}=\sum_{i=1}^{3}\left(\frac{\partial f}{\partial q_{i}} \frac{\partial g}{\partial p_{i}}-\frac{\partial g}{\partial q_{i}} \frac{\partial f}{\partial p_{i}}\right),
$$

turns out to be

$$
\mathcal{T}=\frac{1}{2}\left(p_{r}^{2}+\frac{p_{\theta}^{2}}{\kappa_{2} \mathrm{~S}_{\kappa_{1}}^{2}(r)}+\frac{p_{\phi}^{2}}{\kappa_{2} \mathrm{~S}_{\kappa_{1}}^{2}(r) \mathrm{S}_{\kappa_{2}}^{2}(\theta)}\right) .
$$

Note that the connection (2.13) would allow one to write the geodesic equations whose solution would correspond to the geodesic motion associated with $\mathcal{T}$ (see [8] for the $2 \mathrm{D}$ case). 
Now we proceed to deduce a phase space realization of the Lie generators of $s o_{\kappa_{1}, \kappa_{2}}(4)$. In Weierstrass coordinates $x_{\mu}$ and momenta $p_{\mu}$ this comes from the vector fields (2.10) through the replacement $\partial_{\mu} \rightarrow-p_{\mu}$ :

$$
\begin{array}{ll}
J_{01}=x_{0} p_{1}-\kappa_{1} x_{1} p_{0}, & J_{0 j}=x_{0} p_{j}-\kappa_{1} \kappa_{2} x_{j} p_{0}, \\
J_{23}=x_{2} p_{3}-x_{3} p_{2}, & J_{1 j}=x_{1} p_{j}-\kappa_{2} x_{j} p_{1} .
\end{array}
$$

The metric (2.9) can also be understood as the kinetic energy in the ambient velocities $\dot{x}_{\mu}$ so that the momenta $p_{\mu}$ are $(j=2,3)$ :

$$
p_{0}=\dot{x}_{0} / \kappa_{1}, \quad p_{1}=\dot{x}_{1}, \quad p_{j}=\kappa_{2} \dot{x}_{j}
$$

Next if we compute the velocities $\dot{x}_{i}$ in the parametrization (2.11) and introduce the momenta (3.2) and (3.6) we obtain the relationship between the ambient momenta and the geodesic polar ones:

$$
\begin{aligned}
& p_{0}=-\mathrm{S}_{\kappa_{1}}(r) p_{r}, \\
& p_{1}=\mathrm{C}_{\kappa_{1}}(r) \mathrm{C}_{\kappa_{2}}(\theta) p_{r}-\frac{\mathrm{S}_{\kappa_{2}}(\theta)}{\mathrm{S}_{\kappa_{1}}(r)} p_{\theta}, \\
& p_{2}=\kappa_{2} \mathrm{C}_{\kappa_{1}}(r) \mathrm{S}_{\kappa_{2}}(\theta) \cos \phi p_{r}+\frac{\mathrm{C}_{\kappa_{2}}(\theta) \cos \phi}{\mathrm{S}_{\kappa_{1}}(r)} p_{\theta}-\frac{\sin \phi}{\mathrm{S}_{\kappa_{1}}(r) \mathrm{S}_{\kappa_{2}}(\theta)} p_{\phi}, \\
& p_{3}=\kappa_{2} \mathrm{C}_{\kappa_{1}}(r) \mathrm{S}_{\kappa_{2}}(\theta) \sin \phi p_{r}+\frac{\mathrm{C}_{\kappa_{2}}(\theta) \sin \phi}{\mathrm{S}_{\kappa_{1}}(r)} p_{\theta}+\frac{\cos \phi}{\mathrm{S}_{\kappa_{1}}(r) \mathrm{S}_{\kappa_{2}}(\theta)} p_{\phi} .
\end{aligned}
$$

Hence the generators (3.5) in geodesic polar coordinates and momenta turn out to be

$$
\begin{aligned}
& J_{01}=\mathrm{C}_{\kappa_{2}}(\theta) p_{r}-\frac{\mathrm{S}_{\kappa_{2}}(\theta)}{\mathrm{T}_{\kappa_{1}}(r)} p_{\theta}, \\
& J_{02}=\kappa_{2} \mathrm{~S}_{\kappa_{2}}(\theta) \cos \phi p_{r}+\frac{\mathrm{C}_{\kappa_{2}}(\theta) \cos \phi}{\mathrm{T}_{\kappa_{1}}(r)} p_{\theta}-\frac{\sin \phi}{\mathrm{T}_{\kappa_{1}}(r) \mathrm{S}_{\kappa_{2}}(\theta)} p_{\phi}, \\
& J_{03}=\kappa_{2} \mathrm{~S}_{\kappa_{2}}(\theta) \sin \phi p_{r}+\frac{\mathrm{C}_{\kappa_{2}}(\theta) \sin \phi}{\mathrm{T}_{\kappa_{1}}(r)} p_{\theta}+\frac{\cos \phi}{\mathrm{T}_{\kappa_{1}}(r) \mathrm{S}_{\kappa_{2}}(\theta)} p_{\phi}, \\
& J_{12}=\cos \phi p_{\theta}-\frac{\sin \phi}{\mathrm{T}_{\kappa_{2}}(\theta)} p_{\phi}, \\
& J_{13}=\sin \phi p_{\theta}+\frac{\cos \phi}{\mathrm{T}_{\kappa_{2}}(\theta)} p_{\phi}, \\
& J_{23}=p_{\phi} .
\end{aligned}
$$

By direct computations it can be proven the following statement.

Proposition 1. The generators (3.7) fulfil the commutation relations (2.1) with respect to the Lie-Poisson bracket (3.3) and all of them Poisson commute with $\mathcal{T}$ (3.4).

In this respect, notice that, under (3.7), the kinetic energy is related with the Casimir $\mathcal{C}_{1}(2.2)$ by $2 \kappa_{2} \mathcal{T}=\mathcal{C}_{1}$, while the second Casimir $\mathcal{C}_{2}$ vanishes.

The realization of the generators (3.7) is particularized for each specific space and PoissonLie algebra contained in $\mathbb{S}_{\left[\kappa_{1}\right] \kappa_{2}}^{3}$ and $s o_{\kappa_{1}, \kappa_{2}}(4)$ in Table 2. In order to present the simplest expressions, hereafter we shall set in all the tables $\kappa_{1} \in\{+1,0,-1\}$ and $\kappa_{2} \in\{+1,-1\}$, which corresponds to deal with units $R=\tau=c=1$. 
Table 2. Phase space realization of the generators of $s o_{\kappa_{1}, \kappa_{2}}(4)$ in canonical geodesic polar coordinates and momenta $\left(r, \theta, \phi ; p_{r}, p_{\theta}, p_{\phi}\right)$ on each space $\mathbb{S}_{\left[\kappa_{1}\right] \kappa_{2}}^{3}$ with $\kappa_{1} \in\{+1,0,-1\}$ and $\kappa_{2} \in\{+1,-1\}$.

\begin{tabular}{|c|c|}
\hline 3D Riemannian spaces & $(2+1)$ D Relativistic spacetimes \\
\hline - Spherical space $\mathbb{S}_{[+]+}^{3} \equiv \mathbf{S}^{3}: \quad s o(4)$ & - Anti-de Sitter spacetime $\mathbb{S}_{[+]-}^{3} \equiv \mathbf{A d} \mathbf{S}^{2+1}: \quad s o(2,2)$ \\
\hline$J_{01}=\cos \theta p_{r}-\frac{\sin \theta}{\tan r} p_{\theta}$ & $J_{01}=\cosh \theta p_{r}-\frac{\sinh \theta}{\tan r} p_{\theta}$ \\
\hline$J_{02}=\sin \theta \cos \phi p_{r}+\frac{\cos \theta \cos \phi}{\tan r} p_{\theta}-\frac{\sin \phi p_{\phi}}{\tan r \sin \theta}$ & $J_{02}=-\sinh \theta \cos \phi p_{r}+\frac{\cosh \theta \cos \phi}{\tan r} p_{\theta}-\frac{\sin \phi p_{\phi}}{\tan r \sinh \theta}$ \\
\hline$J_{03}=\sin \theta \sin \phi p_{r}+\frac{\cos \theta \sin \phi}{\tan r} p_{\theta}+\frac{\cos \phi p_{\phi}}{\tan r \sin \theta}$ & $J_{03}=-\sinh \theta \sin \phi p_{r}+\frac{\cosh \theta \sin \phi}{\tan r} p_{\theta}+\frac{\tan r \sinh \theta}{\tan r \sinh \theta}$ \\
\hline$J_{12}=\cos \phi p_{\theta}-\frac{\sin \phi}{\tan \theta} p_{\phi}$ & $J_{12}=\cos \phi p_{\theta}-\frac{\sin \phi}{\tanh \theta} p_{\phi}$ \\
\hline$J_{13}=\sin \phi p_{\theta}+\frac{\cos \phi}{\tan \theta} p_{\phi}$ & $J_{13}=\sin \phi p_{\theta}+\frac{\cos \phi}{\tanh \theta} p_{\phi}$ \\
\hline$J_{23}=p_{\phi}$ & $J_{23}=p_{\phi}$ \\
\hline - Euclidean space $\mathbb{S}_{[0]+}^{3} \equiv \mathbf{E}^{3}: \quad$ iso $(3)$ & - Minkowskian spacetime $\mathbb{S}_{[0]-}^{3} \equiv \mathbf{M}^{2+1}: \quad i s o(2,1)$ \\
\hline$J_{01}=\cos \theta p_{r}-\frac{\sin \theta}{r} p_{\theta}$ & $J_{01}=\cosh \theta p_{r}-\frac{\sinh \theta}{r} p_{\theta}$ \\
\hline$J_{02}=\sin \theta \cos \phi p_{r}+\frac{\cos \theta \cos \phi}{r} p_{\theta}-\frac{\sin \phi}{r \sin \theta} p_{\phi}$ & $J_{02}=-\sinh \theta \cos \phi p_{r}+\frac{\cosh \theta \cos \phi}{r} p_{\theta}-\frac{\sin \phi}{r \sinh \theta} p_{\phi}$ \\
\hline$J_{03}=\sin \theta \sin \phi p_{r}+\frac{\cos \theta \sin \phi}{r} p_{\theta}+\frac{\cos \phi}{r \sin \theta} p_{\phi}$ & $J_{03}=-\sinh \theta \sin \phi p_{r}+\frac{\cosh \theta \sin \phi}{r} p_{\theta}+\frac{\cos \phi}{r \sinh \theta} p_{\phi}$ \\
\hline$J_{12}=\cos \phi p_{\theta}-\frac{\sin \phi}{\tan \theta} p_{\phi}$ & $J_{12}=\cos \phi p_{\theta}-\frac{\sin \phi}{\tanh \theta} p_{\phi}$ \\
\hline$J_{13}=\sin \phi p_{\theta}+\frac{\cos \phi}{\tan \theta} p_{\phi}$ & $J_{13}=\sin \phi p_{\theta}+\frac{\cos \phi}{\tanh \theta} p_{\phi}$ \\
\hline$J_{23}=p_{\phi}$ & $J_{23}=p_{\phi}$ \\
\hline - Hyperbolic space $\mathbb{S}_{[-]+}^{3} \equiv \mathbf{H}^{3}: \quad s o(3,1)$ & - De Sitter spacetime $\mathbb{S}_{[-]-}^{3} \equiv \mathbf{d} \mathbf{S}^{2+1}: \quad s o(3,1)$ \\
\hline$J_{01}=\cos \theta p_{r}-\frac{\sin \theta}{\tanh r} p_{\theta}$ & $J_{01}=\cosh \theta p_{r}-\frac{\sinh \theta}{\tanh r} p_{\theta}$ \\
\hline$J_{02}=\sin \theta \cos \phi p_{r}+\frac{\cos \theta \cos \phi}{\tanh r} p_{\theta}-\frac{\sin \phi p_{\phi}}{\tanh r \sin \theta}$ & $J_{02}=-\sinh \theta \cos \phi p_{r}+\frac{\cosh \theta \cos \phi}{\tanh r} p_{\theta}-\frac{\sin \phi p_{\phi}}{\tanh r \sinh \theta}$ \\
\hline$J_{03}=\sin \theta \sin \phi p_{r}+\frac{\cos \theta \sin \phi}{\tanh r} p_{\theta}+\frac{\cos \phi p_{\phi}}{\tanh r \sin \theta}$ & $J_{03}=-\sinh \theta \sin \phi p_{r}+\frac{\cosh \theta \sin \phi}{\tanh r} p_{\theta}+\frac{\cos \phi p_{\phi}}{\tanh r \sinh \theta}$ \\
\hline$J_{12}=\cos \phi p_{\theta}-\frac{\sin \phi}{\tan \theta} p_{\phi}$ & $J_{12}=\cos \phi p_{\theta}-\frac{\sin \phi}{\tanh \theta} p_{\phi}$ \\
\hline$J_{13}=\sin \phi p_{\theta}+\frac{\cos \phi}{\tan \theta} p_{\phi}$ & $J_{13}=\sin \phi p_{\theta}+\frac{\cos \phi}{\tanh \theta} p_{\phi}$ \\
\hline$J_{23}=p_{\phi}$ & $J_{23}=p_{\phi}$ \\
\hline
\end{tabular}

\section{Superintegrable potentials}

Now if we look for superintegrable potentials $\mathcal{U}(q)=\mathcal{U}(r, \theta, \phi)$ which generalize the Euclidean one (1.1) to the space $\mathbb{S}_{\left[\kappa_{1}\right] \kappa_{2}}^{3}$ we find

$$
\begin{aligned}
\mathcal{U} & =\mathcal{F}^{\prime}\left(x_{0}\right)+\frac{\beta_{1}}{x_{1}^{2}}+\frac{\beta_{2}}{x_{2}^{2}}+\frac{\beta_{3}}{x_{3}^{2}} \\
& =\mathcal{F}(r)+\frac{1}{\mathrm{~S}_{\kappa_{1}}^{2}(r)}\left(\frac{\beta_{1}}{\mathrm{C}_{\kappa_{2}}^{2}(\theta)}+\frac{\beta_{2}}{\mathrm{~S}_{\kappa_{2}}^{2}(\theta) \cos ^{2} \phi}+\frac{\beta_{3}}{\mathrm{~S}_{\kappa_{2}}^{2}(\theta) \sin ^{2} \phi}\right),
\end{aligned}
$$

where $\mathcal{F}^{\prime}\left(\mathrm{C}_{\kappa_{1}}(r)\right) \equiv \mathcal{F}(r)$ is an arbitrary smooth function and $\beta_{i}$ are arbitrary real constants. As in $\mathbf{E}^{3}$, the three $\beta_{i}$-terms can be interpreted on the six spaces in a common way as "centrifugal barriers"; for some particular curved spaces these may admit an alternative interpretation as non-central harmonic oscillators. These facts will be explained in detail in the next section. 
The resulting Hamiltonian $\mathcal{H}=\mathcal{T}+\mathcal{U}$, with kinetic energy (3.4) and potential (4.1), has three integrals of the motion quadratic in the momenta which are associated with the (Lorentz) rotation generators $(j=2,3)$ :

$$
I_{1 j}=J_{1 j}^{2}+2 \beta_{1} \kappa_{2}^{2} \frac{x_{j}^{2}}{x_{1}^{2}}+2 \beta_{j} \kappa_{2} \frac{x_{1}^{2}}{x_{j}^{2}}, \quad I_{23}=J_{23}^{2}+2 \beta_{2} \kappa_{2} \frac{x_{3}^{2}}{x_{2}^{2}}+2 \beta_{3} \kappa_{2} \frac{x_{2}^{2}}{x_{3}^{2}},
$$

which in the geodesic polar phase space explicitly read

$$
\begin{aligned}
& I_{12}=\left(\cos \phi p_{\theta}-\frac{\sin \phi}{\mathrm{T}_{\kappa_{2}}(\theta)} p_{\phi}\right)^{2}+2 \beta_{1} \kappa_{2}^{2} \mathrm{~T}_{\kappa_{2}}^{2}(\theta) \cos ^{2} \phi+\frac{2 \beta_{2} \kappa_{2}}{\mathrm{~T}_{\kappa_{2}}^{2}(\theta) \cos ^{2} \phi}, \\
& I_{13}=\left(\sin \phi p_{\theta}+\frac{\cos \phi}{\mathrm{T}_{\kappa_{2}}(\theta)} p_{\phi}\right)^{2}+2 \beta_{1} \kappa_{2}^{2} \mathrm{~T}_{\kappa_{2}}^{2}(\theta) \sin ^{2} \phi+\frac{2 \beta_{3} \kappa_{2}}{\mathrm{~T}_{\kappa_{2}}^{2}(\theta) \sin ^{2} \phi}, \\
& I_{23}=p_{\phi}^{2}+2 \beta_{2} \kappa_{2} \tan ^{2} \phi+\frac{2 \beta_{3} \kappa_{2}}{\tan ^{2} \phi} .
\end{aligned}
$$

These constants of the motion do not Poisson commute with each other. In order to find quantities in involution we define another integral from the above set:

$$
\begin{aligned}
I_{123} & =I_{12}+I_{13}+\kappa_{2} I_{23}+2 \kappa_{2}\left(\beta_{1}+\kappa_{2} \beta_{2}+\kappa_{2} \beta_{3}\right) \\
& =p_{\theta}^{2}+\frac{p_{\phi}^{2}}{\mathrm{~S}_{\kappa_{2}}^{2}(\theta)}+\frac{2 \beta_{1} \kappa_{2}}{\mathrm{C}_{\kappa_{2}}^{2}(\theta)}+\frac{2 \beta_{2} \kappa_{2}}{\mathrm{~S}_{\kappa_{2}}^{2}(\theta) \cos ^{2} \phi}+\frac{2 \beta_{3} \kappa_{2}}{\mathrm{~S}_{\kappa_{2}}^{2}(\theta) \sin ^{2} \phi},
\end{aligned}
$$

which is related with the Casimir of the rotation subalgebra $\mathfrak{h}_{0}=s o_{\kappa_{2}}(3)$.

Superintegrability of $\mathcal{H}$ is then characterized by:

Proposition 2. (i) The three functions $\left\{I_{12}, I_{123}, \mathcal{H}\right\}$ are mutually in involution. The same holds for the set $\left\{I_{23}, I_{123}, \mathcal{H}\right\}$.

(ii) The four functions $\left\{I_{12}, I_{23}, I_{123}, \mathcal{H}\right\}$ are functionally independent, thus $\mathcal{H}$ is a superintegrable Hamiltonian.

These results, which can be checked directly, are displayed in Table 3 for each particular space arising within $\mathbb{S}_{\left[\kappa_{1}\right] \kappa_{2}}^{3}$. Notice that the integrals $\left\{I_{12}, I_{23}, I_{123}\right\}$ do depend on $\kappa_{2}$ and $\left(\theta, \phi ; p_{\theta}, p_{\phi}\right)$ but neither on the curvature $\kappa_{1}$ nor on $\left(r, p_{r}\right)$, so these are the same for each set of three spaces with the same signature.

A straightforward consequence of the complete integrability determined by $\left\{I_{23}, I_{123}, \mathcal{H}\right\}$ is that $\mathcal{H}$ is separable and we obtain three equations, each of them depending on a canonical pair $\left(q_{i}, p_{i}\right)$ :

$$
\begin{aligned}
& I_{23}\left(\phi, p_{\phi}\right)=p_{\phi}^{2}+2 \beta_{2} \kappa_{2} \tan ^{2} \phi+\frac{2 \beta_{3} \kappa_{2}}{\tan ^{2} \phi}, \\
& I_{123}\left(\theta, p_{\theta}\right)=p_{\theta}^{2}+\frac{2 \beta_{1} \kappa_{2}}{\mathrm{C}_{\kappa_{2}}^{2}(\theta)}+\frac{1}{\mathrm{~S}_{\kappa_{2}}^{2}(\theta)}\left(I_{23}+2 \kappa_{2}\left(\beta_{2}+\beta_{3}\right)\right), \\
& \mathcal{H}\left(r, p_{r}\right)=\frac{1}{2} p_{r}^{2}+\mathcal{F}(r)+\frac{1}{2 \kappa_{2} \mathrm{~S}_{\kappa_{1}}^{2}(r)} I_{123},
\end{aligned}
$$

and $\mathcal{H}$ is so reduced to a $1 \mathrm{D}$ radial system.

Therefore there remains one constant of the motion to obtain maximal superintegrability so that we shall say that $\mathcal{H}$ is a quasi-maximally superintegrable Hamiltonian. In the next sections we study two specific choices for the arbitrary radial function $\mathcal{F}(r)$ that lead to an additional integral thus providing maximally superintegrable potentials. The resulting systems are generalizations of the (curved) harmonic oscillator and $\mathrm{KC}$ potentials with additional terms (dependending on the $\beta_{i}$ ). 
Table 3. Superintegrable Hamiltonian $\mathcal{H}=\mathcal{T}+\mathcal{U}$ and its three constants of the motion $\left\{I_{12}, I_{23}, I_{123}\right\}$ for the six spaces $\mathbb{S}_{\left[\kappa_{1}\right] \kappa_{2}}^{3}$ with $\kappa_{1} \in\{+1,0,-1\}$ and $\kappa_{2} \in\{+1,-1\}$.

3D Riemannian spaces

- Spherical space $\mathbb{S}_{[+]+}^{3} \equiv \mathbf{S}^{3}$

$\mathcal{H}=\frac{1}{2}\left(p_{r}^{2}+\frac{p_{\theta}^{2}}{\sin ^{2} r}+\frac{p_{\phi}^{2}}{\sin ^{2} r \sin ^{2} \theta}\right)+\mathcal{F}(r)+\frac{1}{\sin ^{2} r}\left(\frac{\beta_{1}}{\cos ^{2} \theta}+\frac{\beta_{2}}{\sin ^{2} \theta \cos ^{2} \phi}+\frac{\beta_{3}}{\sin ^{2} \theta \sin ^{2} \phi}\right)$

- Euclidean space $\mathbb{S}_{[0]+}^{3} \equiv \mathbf{E}^{3}$

$\mathcal{H}=\frac{1}{2}\left(p_{r}^{2}+\frac{p_{\theta}^{2}}{r^{2}}+\frac{p_{\phi}^{2}}{r^{2} \sin ^{2} \theta}\right)+\mathcal{F}(r)+\frac{1}{r^{2}}\left(\frac{\beta_{1}}{\cos ^{2} \theta}+\frac{\beta_{2}}{\sin ^{2} \theta \cos ^{2} \phi}+\frac{\beta_{3}}{\sin ^{2} \theta \sin ^{2} \phi}\right)$

- Hyperbolic space $\mathbb{S}_{[-]+}^{3} \equiv \mathbf{H}^{3}$

$\mathcal{H}=\frac{1}{2}\left(p_{r}^{2}+\frac{p_{\theta}^{2}}{\sinh ^{2} r}+\frac{p_{\phi}^{2}}{\sinh ^{2} r \sin ^{2} \theta}\right)+\mathcal{F}(r)+\frac{1}{\sinh ^{2} r}\left(\frac{\beta_{1}}{\cos ^{2} \theta}+\frac{\beta_{2}}{\sin ^{2} \theta \cos ^{2} \phi}+\frac{\beta_{3}}{\sin ^{2} \theta \sin ^{2} \phi}\right)$

$I_{12}=\left(\cos \phi p_{\theta}-\frac{\sin \phi}{\tan \theta} p_{\phi}\right)^{2}+2 \beta_{1} \tan ^{2} \theta \cos ^{2} \phi+\frac{2 \beta_{2}}{\tan ^{2} \theta \cos ^{2} \phi}$

$I_{23}=p_{\phi}^{2}+2 \beta_{2} \tan ^{2} \phi+\frac{2 \beta_{3}}{\tan ^{2} \phi}$

$I_{123}=p_{\theta}^{2}+\frac{p_{\phi}^{2}}{\sin ^{2} \theta}+\frac{2 \beta_{1}}{\cos ^{2} \theta}+\frac{2 \beta_{2}}{\sin ^{2} \theta \cos ^{2} \phi}+\frac{2 \beta_{3}}{\sin ^{2} \theta \sin ^{2} \phi}$

$(2+1)$ D Relativistic spacetimes

- Anti-de Sitter spacetime $\mathbb{S}_{[+]-}^{3} \equiv \mathbf{A d S}^{2+1}$

$\mathcal{H}=\frac{1}{2}\left(p_{r}^{2}-\frac{p_{\theta}^{2}}{\sin ^{2} r}-\frac{p_{\phi}^{2}}{\sin ^{2} r \sinh ^{2} \theta}\right)+\mathcal{F}(r)+\frac{1}{\sin ^{2} r}\left(\frac{\beta_{1}}{\cosh ^{2} \theta}+\frac{\beta_{2}}{\sinh ^{2} \theta \cos ^{2} \phi}+\frac{\beta_{3}}{\sinh ^{2} \theta \sin ^{2} \phi}\right)$

- Minkowskian spacetime $\mathbb{S}_{[0]-}^{3} \equiv \mathbf{M}^{2+1}$

$\mathcal{H}=\frac{1}{2}\left(p_{r}^{2}-\frac{p_{\theta}^{2}}{r^{2}}-\frac{p_{\phi}^{2}}{r^{2} \sinh ^{2} \theta}\right)+\mathcal{F}(r)+\frac{1}{r^{2}}\left(\frac{\beta_{1}}{\cosh ^{2} \theta}+\frac{\beta_{2}}{\sinh ^{2} \theta \cos ^{2} \phi}+\frac{\beta_{3}}{\sinh ^{2} \theta \sin ^{2} \phi}\right)$

- De Sitter spacetime $\mathbb{S}_{[-]-}^{3} \equiv \mathbf{d} \mathbf{S}^{2+1}$

$\mathcal{H}=\frac{1}{2}\left(p_{r}^{2}-\frac{p_{\theta}^{2}}{\sinh ^{2} r}-\frac{p_{\phi}^{2}}{\sinh ^{2} r \sinh ^{2} \theta}\right)+\mathcal{F}(r)+\frac{1}{\sinh ^{2} r}\left(\frac{\beta_{1}}{\cosh ^{2} \theta}+\frac{\beta_{2}}{\sinh ^{2} \theta \cos ^{2} \phi}+\frac{\beta_{3}}{\sinh ^{2} \theta \sin ^{2} \phi}\right)$

$I_{12}=\left(\cos \phi p_{\theta}-\frac{\sin \phi}{\tanh \theta} p_{\phi}\right)^{2}+2 \beta_{1} \tanh ^{2} \theta \cos ^{2} \phi-\frac{2 \beta_{2}}{\tanh ^{2} \theta \cos ^{2} \phi}$

$I_{23}=p_{\phi}^{2}-2 \beta_{2} \tan ^{2} \phi-\frac{2 \beta_{3}}{\tan ^{2} \phi}$

$I_{123}=p_{\theta}^{2}+\frac{p_{\phi}^{2}}{\sinh ^{2} \theta}-\frac{2 \beta_{1}}{\cosh ^{2} \theta}-\frac{2 \beta_{2}}{\sinh ^{2} \theta \cos ^{2} \phi}-\frac{2 \beta_{3}}{\sinh ^{2} \theta \sin ^{2} \phi}$

\section{$5 \quad$ Harmonic oscillator potential}

If we like to extend the (curved) harmonic oscillator potential (1.4) to our six spaces, we have to consider the following choice for the arbitrary function appearing in (4.1):

$$
\mathcal{F}^{\prime}\left(x_{0}\right)=\beta_{0}\left(\frac{1-x_{0}^{2}}{\kappa_{1} x_{0}^{2}}\right)=\beta_{0}\left(\frac{x_{1}^{2}+\kappa_{2} x_{2}^{2}+\kappa_{2} x_{3}^{2}}{x_{0}^{2}}\right), \quad \mathcal{F}(r)=\beta_{0} \mathrm{~T}_{\kappa_{1}}^{2}(r),
$$

where $\beta_{0}$ is an arbitrary real parameter. When the complete Hamiltonian is considered we obtain the generalization of $3 \mathrm{D}$ SW system $(1.2), \mathcal{H}^{\mathrm{SW}}=\mathcal{T}+\mathcal{U}^{\mathrm{SW}}$, to the space $\mathbb{S}_{\left[\kappa_{1}\right] \kappa_{2}}^{3}$, namely

$$
\mathcal{U}^{\mathrm{SW}}=\beta_{0} \mathrm{~T}_{\kappa_{1}}^{2}(r)+\frac{1}{\mathrm{~S}_{\kappa_{1}}^{2}(r)}\left(\frac{\beta_{1}}{\mathrm{C}_{\kappa_{2}}^{2}(\theta)}+\frac{\beta_{2}}{\mathrm{~S}_{\kappa_{2}}^{2}(\theta) \cos ^{2} \phi}+\frac{\beta_{3}}{\mathrm{~S}_{\kappa_{2}}^{2}(\theta) \sin ^{2} \phi}\right) .
$$


As we already mentioned in the introduction, the proper SW Hamiltonian arises in the (flat) Euclidean space $[15,16,17,19]$, here written in polar coordinates, which is formed by an isotropic harmonic oscillator with angular frequency $\omega=\sqrt{\beta_{0}}$ together with three centrifugal barriers associated with the $\beta_{i}$ 's. Different constructions of the SW system on the (curved) spherical and hyperbolic spaces can be found in [9, 20, 23, 28, 30, 31, 39]. More recently such a potential has also been deduced and analysed in the $(1+1) \mathrm{D}$ relativistic spacetimes of constant curvature in $[8,12]$ as well as in 2D spaces of variable curvature in [8].

In our case, any of the translation generators $J_{0 i}(3.7)$ provides a constant of the motion quadratic in the momenta in the form $(j=2,3)$ :

$$
\begin{aligned}
& I_{01}=J_{01}^{2}+2 \beta_{0} \frac{x_{1}^{2}}{x_{0}^{2}}+2 \beta_{1} \frac{x_{1}^{2}}{x_{0}^{2}}, \\
& I_{0 j}=J_{0 j}^{2}+2 \beta_{0} \kappa_{2}^{2} \frac{x_{j}^{2}}{x_{0}^{2}}+2 \beta_{j} \kappa_{2} \frac{x_{0}^{2}}{x_{j}^{2}},
\end{aligned}
$$

that is,

$$
\begin{aligned}
& I_{01}=J_{01}^{2}+2 \beta_{0} \mathrm{~T}_{\kappa_{1}}^{2}(r) \mathrm{C}_{\kappa_{2}}^{2}(\theta)+\frac{2 \beta_{1}}{\mathrm{~T}_{\kappa_{1}}^{2}(r) \mathrm{C}_{\kappa_{2}}^{2}(\theta)}, \\
& I_{02}=J_{02}^{2}+2 \beta_{0} \kappa_{2}^{2} \mathrm{~T}_{\kappa_{1}}^{2}(r) \mathrm{S}_{\kappa_{2}}^{2}(\theta) \cos ^{2} \phi+\frac{2 \beta_{2} \kappa_{2}}{\mathrm{~T}_{\kappa_{1}}^{2}(r) \mathrm{S}_{\kappa_{2}}^{2}(\theta) \cos ^{2} \phi}, \\
& I_{03}=J_{03}^{2}+2 \beta_{0} \kappa_{2}^{2} \mathrm{~T}_{\kappa_{1}}^{2}(r) \mathrm{S}_{\kappa_{2}}^{2}(\theta) \sin ^{2} \phi+\frac{2 \beta_{3} \kappa_{2}}{\mathrm{~T}_{\kappa_{1}}^{2}(r) \mathrm{S}_{\kappa_{2}}^{2}(\theta) \sin ^{2} \phi} .
\end{aligned}
$$

Obviously, the seven integrals of the motion $\left\{I_{01}, I_{02}, I_{03}, I_{12}, I_{23}, I_{123}, \mathcal{H}^{\mathrm{SW}}\right\}$ cannot be functionally independent. One constraint for them is given by

$$
2 \kappa_{2} \mathcal{H}^{\mathrm{SW}}=\kappa_{2} I_{01}+I_{02}+I_{03}+\kappa_{1} I_{123},
$$

which reminds the aforementioned relation for the geodesic motion $2 \kappa_{2} \mathcal{T}=\mathcal{C}_{1}$. Note also that

$$
\left\{I_{01}, I_{23}\right\}=\left\{I_{02}, I_{13}\right\}=\left\{I_{03}, I_{12}\right\}=0 .
$$

The final result concerning the superintegrability of $\mathcal{H}^{\mathrm{SW}}$ is established by:

Proposition 3. (i) Each function $I_{0 i}(5.4)(i=1,2,3)$ Poisson commutes with $\mathcal{H}^{\mathrm{SW}}$.

(ii) The five functions $\left\{I_{0 i}, I_{12}, I_{23}, I_{123}, \mathcal{H}^{\mathrm{SW}}\right\}$, where $i$ is fixed, are functionally independent, thus $\mathcal{H}^{\mathrm{SW}}$ is a maximally superintegrable Hamiltonian.

The Hamiltonian $\mathcal{H}^{\mathrm{SW}}$ and the additional constant of the motion $I_{01}$ (that ensures maximal superintegrability) are presented for each particular space contained in $\mathbb{S}_{\left[\kappa_{1}\right] \kappa_{2}}^{3}$ in Table 4.

\subsection{Description of the SW potential}

The $2 \mathrm{D}$ version of the potential $\mathcal{U}^{\mathrm{SW}}(5.2)$ on $\mathbf{S}^{2}$ has been interpreted in $[40,41,42]$ as a superposition of three spherical oscillators; the interpretation for arbitrary dimension on $\mathbf{S}^{N}$ and $\mathbf{H}^{N}$ has been presented in [9, 23]. Furthermore, a detail description on this potential on $\mathbf{A d} \mathbf{S}^{1+1}$, $\mathbf{M}^{1+1}$ and $\mathbf{d} \mathbf{S}^{1+1}$ was recently performed in [8]. In what follows we analyse the (physical) geometrical role of the $3 \mathrm{D}$ potential (5.2) on each particular space $\mathbb{S}_{\left[\kappa_{1}\right] \kappa_{2}}^{3}$ thus generalizing all of the mentioned $2 \mathrm{D}$ results. 
Table 4. Maximally superintegrable Smorodinsky-Winternitz Hamiltonian $\mathcal{H}^{\mathrm{SW}}=\mathcal{T}+\mathcal{U}^{\mathrm{SW}}$ and the additional constant of the motion $I_{01}$ to the set $\left\{I_{12}, I_{23}, I_{123}\right\}$ for the six spaces $\mathbb{S}_{\left[\kappa_{1}\right] \kappa_{2}}^{3}$ with the same conventions given in Table 3 .

3D Riemannian spaces

- Spherical space $\mathbf{S}^{3}$

$\mathcal{H}^{\mathrm{SW}}=\frac{1}{2}\left(p_{r}^{2}+\frac{p_{\theta}^{2}}{\sin ^{2} r}+\frac{p_{\phi}^{2}}{\sin ^{2} r \sin ^{2} \theta}\right)+\beta_{0} \tan ^{2} r+\frac{1}{\sin ^{2} r}\left(\frac{\beta_{1}}{\cos ^{2} \theta}+\frac{\beta_{2}}{\sin ^{2} \theta \cos ^{2} \phi}+\frac{\beta_{3}}{\sin ^{2} \theta \sin ^{2} \phi}\right)$

$I_{01}=\left(\cos \theta p_{r}-\frac{\sin \theta}{\tan r} p_{\theta}\right)^{2}+2 \beta_{0} \tan ^{2} r \cos ^{2} \theta+\frac{2 \beta_{1}}{\tan ^{2} r \cos ^{2} \theta}$

- Euclidean space $\mathbf{E}^{3}$

$\mathcal{H}^{\mathrm{SW}}=\frac{1}{2}\left(p_{r}^{2}+\frac{p_{\theta}^{2}}{r^{2}}+\frac{p_{\phi}^{2}}{r^{2} \sin ^{2} \theta}\right)+\beta_{0} r^{2}+\frac{1}{r^{2}}\left(\frac{\beta_{1}}{\cos ^{2} \theta}+\frac{\beta_{2}}{\sin ^{2} \theta \cos ^{2} \phi}+\frac{\beta_{3}}{\sin ^{2} \theta \sin ^{2} \phi}\right)$

$I_{01}=\left(\cos \theta p_{r}-\frac{\sin \theta}{r} p_{\theta}\right)^{2}+2 \beta_{0} r^{2} \cos ^{2} \theta+\frac{2 \beta_{1}}{r^{2} \cos ^{2} \theta}$

- Hyperbolic space $\mathbf{H}^{3}$

$$
\begin{aligned}
& \mathcal{H}^{\mathrm{SW}}=\frac{1}{2}\left(p_{r}^{2}+\frac{p_{\theta}^{2}}{\sinh ^{2} r}+\frac{p_{\phi}^{2}}{\sinh ^{2} r \sin ^{2} \theta}\right)+\beta_{0} \tanh ^{2} r+\frac{1}{\sinh ^{2} r}\left(\frac{\beta_{1}}{\cos ^{2} \theta}+\frac{\beta_{2}}{\sin ^{2} \theta \cos ^{2} \phi}+\frac{\beta_{3}}{\sin ^{2} \theta \sin ^{2} \phi}\right) \\
& I_{01}=\left(\cos \theta p_{r}-\frac{\sin \theta}{\tanh r} p_{\theta}\right)^{2}+2 \beta_{0} \tanh ^{2} r \cos ^{2} \theta+\frac{2 \beta_{1}}{\tanh ^{2} r \cos ^{2} \theta}
\end{aligned}
$$

$(2+1)$ D Relativistic spacetimes

- Anti-de Sitter spacetime $\mathbf{A d} \mathbf{S}^{2+1}$

$\mathcal{H}^{\mathrm{SW}}=\frac{1}{2}\left(p_{r}^{2}-\frac{p_{\theta}^{2}}{\sin ^{2} r}-\frac{p_{\phi}^{2}}{\sin ^{2} r \sinh ^{2} \theta}\right)+\beta_{0} \tan ^{2} r+\frac{1}{\sin ^{2} r}\left(\frac{\beta_{1}}{\cosh ^{2} \theta}+\frac{\beta_{2}}{\sinh ^{2} \theta \cos ^{2} \phi}+\frac{\beta_{3}}{\sinh ^{2} \theta \sin ^{2} \phi}\right)$

$I_{01}=\left(\cosh \theta p_{r}-\frac{\sinh \theta}{\tan r} p_{\theta}\right)^{2}+2 \beta_{0} \tan ^{2} r \cosh ^{2} \theta+\frac{2 \beta_{1}}{\tan ^{2} r \cosh ^{2} \theta}$

- Minkowskian spacetime $\mathbf{M}^{2+1}$

$$
\begin{aligned}
& \mathcal{H}^{\mathrm{SW}}=\frac{1}{2}\left(p_{r}^{2}-\frac{p_{\theta}^{2}}{r^{2}}-\frac{p_{\phi}^{2}}{r^{2} \sinh ^{2} \theta}\right)+\beta_{0} r^{2}+\frac{1}{r^{2}}\left(\frac{\beta_{1}}{\cosh ^{2} \theta}+\frac{\beta_{2}}{\sinh ^{2} \theta \cos ^{2} \phi}+\frac{\beta_{3}}{\sinh ^{2} \theta \sin ^{2} \phi}\right) \\
& I_{01}=\left(\cosh \theta p_{r}-\frac{\sinh \theta}{r} p_{\theta}\right)^{2}+2 \beta_{0} r^{2} \cosh ^{2} \theta+\frac{2 \beta_{1}}{r^{2} \cosh ^{2} \theta}
\end{aligned}
$$

- De Sitter spacetime $\mathbf{d} \mathbf{S}^{2+1}$

$$
\begin{aligned}
& \mathcal{H}^{\mathrm{SW}}=\frac{1}{2}\left(p_{r}^{2}-\frac{p_{\theta}^{2}}{\sinh ^{2} r}-\frac{p_{\phi}^{2}}{\sinh ^{2} r \sinh ^{2} \theta}\right)+\beta_{0} \tanh ^{2} r+\frac{1}{\sinh ^{2} r}\left(\frac{\beta_{1}}{\cosh ^{2} \theta}+\frac{\beta_{2}}{\sinh ^{2} \theta \cos ^{2} \phi}+\frac{\beta_{3}}{\sinh ^{2} \theta \sin ^{2} \phi}\right) \\
& I_{01}=\left(\cosh \theta p_{r}-\frac{\sinh \theta}{\tanh r} p_{\theta}\right)^{2}+2 \beta_{0} \tanh ^{2} r \cosh ^{2} \theta+\frac{2 \beta_{1}}{\tanh ^{2} r \cosh ^{2} \theta}
\end{aligned}
$$

Consider the (time-like) geodesic $l_{1}$ and the two (space-like) geodesics $l_{2}, l_{3}$ in $\mathbb{S}_{\left[\kappa_{1}\right] \kappa_{2}}^{3}$ orthogonal at the origin $O$ and the generic point $Q(r, \theta, \phi)$ as given in Subsection 2.2. Next let $Q_{i j}$ $(i, j=1,2,3 ; i<j)$ be the intersection point of the reference flag spanned by $l_{i}$ and $l_{j}$ (the 2-plane $l_{i} l_{j}$ ) with its orthogonal geodesic through $Q$. Hence we introduce the (time-like) geodesic distance $x=Q Q_{23}$ and the two (space-like) distances $y=Q Q_{13}, z=Q Q_{12}$. Finally, let $Q_{1}$ be the intersection point of $l_{1}$ with its orthogonal (space-like) geodesic $l_{1}^{\prime}$ through $Q$ for which $h=Q Q_{1}$ is the (space-like) distance measured along $l_{1}^{\prime}$. Now by applying trigonometry [24] on the orthogonal triangles $O Q Q_{1}$ (with inner angle $\theta$ ), $O Q Q_{23}$ (with external angle $\theta$ ), $Q_{13} Q Q_{1}$ (with external angle $\phi$ ) and $Q_{12} Q Q_{1}$ (with inner angle $\phi$ ), we find that

$$
\begin{array}{ll}
O Q Q_{1}: & \mathrm{S}_{\kappa_{1} \kappa_{2}}(h)=\mathrm{S}_{\kappa_{1}}(r) \mathrm{S}_{\kappa_{2}}(\theta), \\
O Q Q_{23}: & \mathrm{S}_{\kappa_{1}}(x)=\mathrm{S}_{\kappa_{1}}(r) \mathrm{C}_{\kappa_{2}}(\theta),
\end{array}
$$




$$
\begin{array}{ll}
Q_{13} Q Q_{1}: & \mathrm{S}_{\kappa_{1} \kappa_{2}}(y)=\mathrm{S}_{\kappa_{1} \kappa_{2}}(h) \cos \phi \\
Q_{12} Q Q_{1}: & \mathrm{S}_{\kappa_{1} \kappa_{2}}(z)=\mathrm{S}_{\kappa_{1} \kappa_{2}}(h) \sin \phi .
\end{array}
$$

Hence the ambient coordinates $x_{i}(2.11)$ can be expressed as

$$
\begin{aligned}
& x_{1}=\mathrm{S}_{\kappa_{1}}(r) \mathrm{C}_{\kappa_{2}}(\theta)=\mathrm{S}_{\kappa_{1}}(x), \\
& x_{2}=\mathrm{S}_{\kappa_{1}}(r) \mathrm{S}_{\kappa_{2}}(\theta) \cos \phi=\mathrm{S}_{\kappa_{1} \kappa_{2}}(y), \\
& x_{3}=\mathrm{S}_{\kappa_{1}}(r) \mathrm{S}_{\kappa_{2}}(\theta) \sin \phi=\mathrm{S}_{\kappa_{1} \kappa_{2}}(z),
\end{aligned}
$$

so that the SW potential (5.2) can be rewritten as

$$
\mathcal{U}^{\mathrm{SW}}=\beta_{0} \mathrm{~T}_{\kappa_{1}}^{2}(r)+\frac{\beta_{1}}{\mathrm{~S}_{\kappa_{1}}^{2}(x)}+\frac{\beta_{2}}{\mathrm{~S}_{\kappa_{1} \kappa_{2}}^{2}(y)}+\frac{\beta_{3}}{\mathrm{~S}_{\kappa_{1} \kappa_{2}}^{2}(z)},
$$

which allows for a unified interpretation on the six spaces:

- The $\beta_{0}$-term is a central harmonic oscillator, that is, the Higgs oscillator [26] with center at the origin $O$.

- The three $\beta_{i}$-terms $(i=1,2,3)$ are "centrifugal barriers".

Furthermore, the $\beta_{i}$-potentials can be interpreted as non-central oscillators in some particular spaces that we proceed to describe by considering the simplest values for $\kappa_{i} \in\{ \pm 1\}$.

\subsubsection{Spherical space $\mathrm{S}^{3}$}

Let $O_{i}$ be the points placed along the basic geodesics $l_{i}(i=1,2,3)$ which are a quadrant apart from the origin $O$, that is, each two points taken from the set $\left\{O, O_{i}\right\}$ are mutually separated a distance $\frac{\pi}{2}$ (if $\kappa_{1}=1 / R^{2}$, a quadrant is $\pi /\left(2 \sqrt{\kappa_{1}}\right)=R \pi / 2$ ). In fact, each $O_{i}$ is the intersection point between the geodesic $l_{i}$ and the axis $x_{i}$ of the ambient space. If we denote by $r_{i}$ the distance between $Q$ and $O_{i}$ measured along the geodesic joining both points then

$$
r_{1}+x=r_{2}+y=r_{3}+z=\frac{\pi}{2},
$$

which means that each set of three points $\left\{O_{1} Q Q_{23}\right\},\left\{O_{2} Q Q_{13}\right\}$ and $\left\{O_{3} Q Q_{12}\right\}$ lie on the same geodesic. Thus

$$
x_{1}=\sin x=\cos r_{1}, \quad x_{2}=\sin y=\cos r_{2}, \quad x_{3}=\sin z=\cos r_{3},
$$

so that the SW potential (5.2) on $\mathbf{S}^{3}$ can be expressed in two manners

$$
\begin{aligned}
\mathcal{U}^{\mathrm{SW}} & =\beta_{0} \tan ^{2} r+\frac{\beta_{1}}{\sin ^{2} x}+\frac{\beta_{2}}{\sin ^{2} y}+\frac{\beta_{3}}{\sin ^{2} z} \\
& =\beta_{0} \tan ^{2} r+\sum_{i=1}^{3}\left(\beta_{i} \tan ^{2} r_{i}+\beta_{i}\right),
\end{aligned}
$$

which show a superposition of the central spherical oscillator with center at $O$ either with three spherical centrifugal barriers, or with three spherical oscillators with centers placed at $O_{i}[9,23]$.

\subsubsection{Hyperbolic space $\mathbf{H}^{3}$}

The analogous points to the previous "centers" $O_{i}$ would be beyond the "actual" hyperbolic space and so placed in the exterior ("ideal") region of $\mathbf{H}^{3}$. The SW potential can only written in the form (5.6):

$$
\mathcal{U}^{\mathrm{SW}}=\beta_{0} \tanh ^{2} r+\frac{\beta_{1}}{\sinh ^{2} x}+\frac{\beta_{2}}{\sinh ^{2} y}+\frac{\beta_{3}}{\sinh ^{2} z},
$$

giving rise to the superposition of a central hyperbolic oscillator with three hyperbolic centrifugal barriers [23]. 


\subsubsection{Euclidean space $\mathbf{E}^{3}$}

The contraction $\kappa_{1} \rightarrow 0(R \rightarrow \infty)$ of the SW potential on $\mathbf{S}^{3}$ and $\mathbf{H}^{3}$ can be applied on both expressions (5.7) and (5.9) reducing to

$$
\mathcal{U}^{\mathrm{SW}}=\beta_{0} r^{2}+\frac{\beta_{1}}{x^{2}}+\frac{\beta_{2}}{y^{2}}+\frac{\beta_{3}}{z^{2}},
$$

which is just the proper SW potential (1.2) formed by the flat harmonic oscillator with three centrifugal barriers; in this case $(x, y, z)$ are Cartesian coordinates on $\mathbf{E}^{3}$ and $r^{2}=x^{2}+y^{2}+z^{2}$. This contraction cannot be performed on $\mathbf{S}^{3}$ when the potential is written in the form (5.8); notice that if $\kappa_{1} \rightarrow 0$ the points $O_{i} \rightarrow \infty$.

\subsubsection{Anti-de Sitter spacetime $\operatorname{AdS}^{2+1}$}

We consider the intersection point $O_{1}$ between the time-like geodesic $l_{1}$ and the axis $x_{1}$ of the ambient space, which is at a time-like distance $\frac{\pi}{2}$ from the origin $O$ [8]. If $r_{1}$ denotes the time-like distance $Q O_{1}$, then $r_{1}+x=\frac{\pi}{2}$. Therefore the SW potential becomes

$$
\begin{aligned}
\mathcal{U}^{\mathrm{SW}} & =\beta_{0} \tan ^{2} r+\frac{\beta_{1}}{\sin ^{2} x}+\frac{\beta_{2}}{\sinh ^{2} y}+\frac{\beta_{3}}{\sinh ^{2} z} \\
& =\beta_{0} \tan ^{2} r+\beta_{1} \tan ^{2} r_{1}+\frac{\beta_{2}}{\sinh ^{2} y}+\frac{\beta_{3}}{\sinh ^{2} z}+\beta_{1} .
\end{aligned}
$$

The former expression corresponds to the superposition of a time-like (spherical) oscillator centered at $O$ with a time-like (spherical) centrifugal potential and two space-like (hyperbolic) ones. Under the latter form, the time-like centrifugal term is transformed into another spherical oscillator now with center at $O_{1}$.

\subsubsection{De Sitter spacetime $\mathrm{dS}^{2+1}$}

Recall that $\mathbf{A d} \mathbf{S}^{2+1}$ and $\mathbf{d} \mathbf{S}^{2+1}$ are related through an interchange between time-like lines and space-like ones; the former are compact (circular) on $\mathbf{A d} \mathbf{S}^{2+1}$ and non-compact (hyperbolic) on $\mathbf{d} \mathbf{S}^{2+1}$, while the latter are non-compact on $\mathbf{A} \mathbf{d} \mathbf{S}^{2+1}$ but compact on $\mathbf{d} \mathbf{S}^{2+1}$.

So, we consider the intersection point $O_{j}(j=2,3)$ between the space-like geodesic $l_{j}$ and the axis $x_{j}$ which is at a space-like distance $\frac{\pi}{2}$ from $O$, so that $r_{j}$ is the space-like distance $Q O_{j}$ verifying $r_{2}+y=r_{3}+z=\frac{\pi}{2}$ [8]. Hence the SW potential can be rewritten as

$$
\begin{aligned}
\mathcal{U}^{\mathrm{SW}} & =\beta_{0} \tanh ^{2} r+\frac{\beta_{1}}{\sinh ^{2} x}+\frac{\beta_{2}}{\sin ^{2} y}+\frac{\beta_{3}}{\sin ^{2} z} \\
& =\beta_{0} \tanh ^{2} r+\frac{\beta_{1}}{\sinh ^{2} x}+\beta_{2} \tan ^{2} r_{2}+\beta_{3} \tan ^{2} r_{3}+\beta_{2}+\beta_{3} .
\end{aligned}
$$

In this way, we find the superposition of a central time-like (hyperbolic) oscillator with a timelike (hyperbolic) centrifugal barrier, and either with two other space-like (spherical) centrifugal barriers or with two space-like (spherical) oscillators centered at $O_{j}$.

\subsubsection{Minkowskian spacetime $\mathrm{M}^{2+1}$}

Finally, the contraction $\kappa_{1} \rightarrow 0(\tau \rightarrow \infty)$ of (5.11) and (5.13) gives

$$
\mathcal{U}^{\mathrm{SW}}=\beta_{0} r^{2}+\frac{\beta_{1}}{x^{2}}+\frac{\beta_{2}}{y^{2}}+\frac{\beta_{3}}{z^{2}}
$$


which is formed by a time-like harmonic oscillator $\beta_{0} r^{2}$, one time-like centrifugal barrier $\beta_{1} / x^{2}$ together with two space-like ones $\beta_{2} / y^{2}, \beta_{3} / z^{2}$. The coordinates $(x, y, z)$ are the usual time and space ones such that $r^{2}=x^{2}-y^{2}-z^{2}$. On the contrary, the expressions (5.12) and (5.14) are not well defined when $\kappa_{1} \rightarrow 0$ since the points $O_{1}$ and $O_{j}$ go to infinity.

\section{Kepler-Coulomb potential}

The generalization of the KC potential (1.4) to the space $\mathbb{S}_{\left[\kappa_{1}\right] \kappa_{2}}^{3}$ is achieved by choosing

$$
\mathcal{F}^{\prime}\left(x_{0}\right)=-k \frac{x_{0}}{\sqrt{\left(1-x_{0}^{2}\right) / \kappa_{1}}}=-k \frac{x_{0}}{\sqrt{x_{1}^{2}+\kappa_{2} x_{2}^{2}+\kappa_{2} x_{3}^{2}}}, \quad \mathcal{F}(r)=-\frac{k}{\mathrm{~T}_{\kappa_{1}}(r)},
$$

where $k$ is an arbitrary real parameter. As it already happens in $\mathbf{E}^{3}$ [14], it is not possible to add the three potential terms depending on the $\beta_{i}$ 's keeping at the same time maximal superintegrability; so that, at least, one of them must vanish. Consequently, we find, in principle, three possible generalizations of the Euclidean potential (1.3) to $\mathbb{S}_{\left[\kappa_{1}\right] \kappa_{2}}^{3}$ :

$$
\begin{aligned}
& \mathcal{U}_{1}^{\mathrm{GKC}}=-\frac{k}{\mathrm{~T}_{\kappa_{1}}(r)}+\frac{1}{\mathrm{~S}_{\kappa_{1}}^{2}(r) \mathrm{S}_{\kappa_{2}}^{2}(\theta)}\left(\frac{\beta_{2}}{\cos ^{2} \phi}+\frac{\beta_{3}}{\sin ^{2} \phi}\right), \\
& \mathcal{U}_{2}^{\mathrm{GKC}}=-\frac{k}{\mathrm{~T}_{\kappa_{1}}(r)}+\frac{1}{\mathrm{~S}_{\kappa_{1}}^{2}(r)}\left(\frac{\beta_{1}}{\mathrm{C}_{\kappa_{2}}^{2}(\theta)}+\frac{\beta_{3}}{\mathrm{~S}_{\kappa_{2}}^{2}(\theta) \sin ^{2} \phi}\right), \\
& \mathcal{U}_{3}^{\mathrm{GKC}}=-\frac{k}{\mathrm{~T}_{\kappa_{1}}(r)}+\frac{1}{\mathrm{~S}_{\kappa_{1}}^{2}(r)}\left(\frac{\beta_{1}}{\mathrm{C}_{\kappa_{2}}^{2}(\theta)}+\frac{\beta_{2}}{\mathrm{~S}_{\kappa_{2}}^{2}(\theta) \cos ^{2} \phi}\right) .
\end{aligned}
$$

Thus each potential $\mathcal{U}_{i}^{\mathrm{GKC}}$ contains the proper $\mathrm{KC} k$-term $[11,12,28,31,32,33,37,39,43]$ together with two additional $\beta_{i}$-terms, which can further be interpreted as centrifugal barriers or non-central oscillators; for each of them there is an additional constant of the motion given by $(i=1,2,3)$ :

$$
L_{i}=\sum_{l=1 ; l \neq i}^{3} J_{0 l} J_{l i}+k \frac{\kappa_{2} x_{i}}{\sqrt{x_{1}^{2}+\kappa_{2} x_{2}^{2}+\kappa_{2} x_{3}^{2}}}-2 \kappa_{2} \sum_{l=1 ; l \neq i}^{3} \beta_{l} \frac{x_{0} x_{i}}{x_{l}^{2}}
$$

where $J_{l i}=-J_{i l}$ for $i<l$. In terms of the geodesic polar phase space these integrals read

$$
\begin{aligned}
& L_{1}=-J_{02} J_{12}-J_{03} J_{13}+k \kappa_{2} \mathrm{C}_{\kappa_{2}}(\theta)-\frac{2 \kappa_{2} \mathrm{C}_{\kappa_{2}}(\theta)}{\mathrm{T}_{\kappa_{1}}(r) \mathrm{S}_{\kappa_{2}}^{2}(\theta)}\left(\frac{\beta_{2}}{\cos ^{2} \phi}+\frac{\beta_{3}}{\sin ^{2} \phi}\right), \\
& L_{2}=J_{01} J_{12}-J_{03} J_{23}+k \kappa_{2} \mathrm{~S}_{\kappa_{2}}(\theta) \cos \phi-\frac{2 \kappa_{2} \cos \phi}{\mathrm{T}_{\kappa_{1}}(r)}\left(\frac{\beta_{1} \mathrm{~S}_{\kappa_{2}}(\theta)}{\mathrm{C}_{\kappa_{2}}^{2}(\theta)}+\frac{\beta_{3}}{\mathrm{~S}_{\kappa_{2}}(\theta) \sin ^{2} \phi}\right), \\
& L_{3}=J_{01} J_{13}+J_{02} J_{23}+k \kappa_{2} \mathrm{~S}_{\kappa_{2}}(\theta) \sin \phi-\frac{2 \kappa_{2} \sin \phi}{\mathrm{T}_{\kappa_{1}}(r)}\left(\frac{\beta_{1} \mathrm{~S}_{\kappa_{2}}(\theta)}{\mathrm{C}_{\kappa_{2}}^{2}(\theta)}+\frac{\beta_{2}}{\mathrm{~S}_{\kappa_{2}}(\theta) \cos ^{2} \phi}\right) .
\end{aligned}
$$

The superintegrability of each Hamiltonian $\mathcal{H}_{i}^{\mathrm{GKC}}=\mathcal{T}+\mathcal{U}_{i}^{\mathrm{GKC}}(i=1,2,3)$ is determined by:

Proposition 4. (i) The function $L_{i}$ (6.4) Poisson commutes with $\mathcal{H}_{i}^{\mathrm{GKC}}$.

(ii) The five functions $\left\{L_{i}, I_{12}, I_{23}, I_{123}, \mathcal{H}_{i}^{\mathrm{GKC}}\right\}$ are functionally independent, thus $\mathcal{H}_{i}^{\mathrm{GKC}}$ is a maximally superintegrable Hamiltonian. 


\subsection{The Laplace-Runge-Lenz vector}

When another $\beta_{j}$ is taken equal to zero in a given potential $\mathcal{U}_{i}^{\mathrm{GKC}}(j \neq i)$, the function $L_{j}$ is also a constant of the motion. Therefore when all the $\beta_{j}=0$, the three functions (6.4) are constants of the motion for the GKC potential which reduces in this case to the proper KC system. This is summed up in the following statements.

Proposition 5. Let one $\beta_{j}=0$ in the Hamiltonian $\mathcal{H}_{i}^{\mathrm{GKC}}=\mathcal{T}+\mathcal{U}_{i}^{\mathrm{GKC}}(i=1,2,3)$ with $j \neq i$.

(i) The two functions $L_{i}, L_{j}$ Poisson commute with $\mathcal{H}_{i}^{\mathrm{GKC}}$.

(ii) The functions $\left\{I_{12}, I_{23}, I_{123}, \mathcal{H}_{i}^{\mathrm{GKC}}\right\}$ together with either $L_{i}$ or $L_{j}$ are functionally independent.

Proposition 6. Let the three $\beta_{i}=0$, then:

(i) The three GKC potentials reduce to its common $k$-term, $\mathcal{U}_{i}^{\mathrm{GKC}} \equiv \mathcal{U}^{\mathrm{KC}}=-k / \mathrm{T}_{\kappa_{1}}(r)$, which is the (curved) KC potential on $\mathbb{S}_{\left[\kappa_{1}\right] \kappa_{2}}^{3}$.

(ii) The three functions

$$
\begin{aligned}
& L_{1}=-J_{02} J_{12}-J_{03} J_{13}+k \kappa_{2} \mathrm{C}_{\kappa_{2}}(\theta), \\
& L_{2}=J_{01} J_{12}-J_{03} J_{23}+k \kappa_{2} \mathrm{~S}_{\kappa_{2}}(\theta) \cos \phi, \\
& L_{3}=J_{01} J_{13}+J_{02} J_{23}+k \kappa_{2} \mathrm{~S}_{\kappa_{2}}(\theta) \sin \phi,
\end{aligned}
$$

Poisson commutes with $\mathcal{H}^{\mathrm{KC}}=\mathcal{T}+\mathcal{U}^{\mathrm{KC}}$ and these are the components of the Laplace-RungeLenz vector on $\mathbb{S}_{\left[\kappa_{1}\right] \kappa_{2}}^{3}$.

(iii) The functions $\left\{I_{12}, I_{23}, I_{123}, \mathcal{H}_{i}^{\mathrm{GKC}}\right\}$ together with any of the components $L_{i}$ are functionally independent.

On the other hand, equivalence amongst the Hamiltonians $\mathcal{H}_{i}^{\mathrm{GKC}}$ comes from their interpretation on $\mathbb{S}_{\left[\kappa_{1}\right] \kappa_{2}}^{3}$ that we proceed to study. We shall show that the three potentials (6.2) are equivalent on the Riemannian spaces (take $i=3$ ), meanwhile we can distinguish two different potentials on the spacetimes (take $i=1,3$ ). Thus we display in Table 5 the corresponding non-equivalent GKC potentials together with the additional constant of the motion (6.4).

\subsection{Description of the GKC potential}

In Subsection 5.1 we have interpreted each of the $\beta_{i}$-terms appearing within the SW potential either as a non-central oscillator or as a centrifugal barrier according to the particular space under consideration. This, in turn, means that each potential (6.2) is a superposition of the KC potential with either oscillators or centrifugal barriers. The latter interpretation arises directly by introducing the distances $(x, y, z)(5.5)$ and this holds simultaneously for the six spaces:

$$
\begin{aligned}
& \mathcal{U}_{1}^{\mathrm{GKC}}=-\frac{k}{\mathrm{~T}_{\kappa_{1}}(r)}+\frac{\beta_{2}}{\mathrm{~S}_{\kappa_{1} \kappa_{2}}^{2}(y)}+\frac{\beta_{3}}{\mathrm{~S}_{\kappa_{1} \kappa_{2}}^{2}(z)}, \\
& \mathcal{U}_{2}^{\mathrm{GKC}}=-\frac{k}{\mathrm{~T}_{\kappa_{1}}(r)}+\frac{\beta_{1}}{\mathrm{~S}_{\kappa_{1}}^{2}(x)}+\frac{\beta_{3}}{\mathrm{~S}_{\kappa_{1} \kappa_{2}}^{2}(z)}, \\
& \mathcal{U}_{3}^{\mathrm{GKC}}=-\frac{k}{\mathrm{~T}_{\kappa_{1}}(r)}+\frac{\beta_{1}}{\mathrm{~S}_{\kappa_{1}}^{2}(x)}+\frac{\beta_{2}}{\mathrm{~S}_{\kappa_{1} \kappa_{2}}^{2}(y)} .
\end{aligned}
$$

These expressions clearly show that some $\mathcal{H}_{i}^{\mathrm{GKC}}$ are equivalent according to the value of $\kappa_{2}$, that is, the signature of the metric, so that we analyze the two possibilities separately. 
Table 5. Maximally superintegrable generalized Kepler-Coulomb potential $\mathcal{U}_{i}^{\mathrm{GKC}}$, such that $\mathcal{H}_{i}^{\mathrm{GKC}}=\mathcal{T}+\mathcal{U}_{i}^{\mathrm{GKC}}$, and the additional constant of the motion $L_{i}$ to the set $\left\{I_{12}, I_{23}, I_{123}\right\}$ for $\mathbb{S}_{\left[\kappa_{1}\right] \kappa_{2}}^{3}$ with the same conventions given in Table 3 ( $i=3$ for the Riemannian spaces and $i=3,1$ for the spacetimes).

3D Riemannian spaces

- Spherical space $\mathbf{S}^{3}$

$\mathcal{U}_{3}^{\mathrm{GKC}}=-\frac{k}{\tan r}+\frac{1}{\sin ^{2} r}\left(\frac{\beta_{1}}{\cos ^{2} \theta}+\frac{\beta_{2}}{\sin ^{2} \theta \cos ^{2} \phi}\right)$

$L_{3}=J_{01} J_{13}+J_{02} J_{23}+k \sin \theta \sin \phi-\frac{2 \sin \phi}{\tan r}\left(\frac{\beta_{1} \sin \theta}{\cos ^{2} \theta}+\frac{\beta_{2}}{\sin \theta \cos ^{2} \phi}\right)$

- Euclidean space $\mathbf{E}^{3}$

$\mathcal{U}_{3}^{\mathrm{GKC}}=-\frac{k}{r}+\frac{1}{r^{2}}\left(\frac{\beta_{1}}{\cos ^{2} \theta}+\frac{\beta_{2}}{\sin ^{2} \theta \cos ^{2} \phi}\right)$

$L_{3}=J_{01} J_{13}+J_{02} J_{23}+k \sin \theta \sin \phi-\frac{2 \sin \phi}{r}\left(\frac{\beta_{1} \sin \theta}{\cos ^{2} \theta}+\frac{\beta_{2}}{\sin \theta \cos ^{2} \phi}\right)$

- Hyperbolic space $\mathbf{H}^{3}$

$$
\begin{aligned}
& \mathcal{U}_{3}^{\mathrm{GKC}}=-\frac{k}{\tanh r}+\frac{1}{\sinh ^{2} r}\left(\frac{\beta_{1}}{\cos ^{2} \theta}+\frac{\beta_{2}}{\sin ^{2} \theta \cos ^{2} \phi}\right) \\
& L_{3}=J_{01} J_{13}+J_{02} J_{23}+k \sin \theta \sin \phi-\frac{2 \sin \phi}{\tanh r}\left(\frac{\beta_{1} \sin \theta}{\cos ^{2} \theta}+\frac{\beta_{2}}{\sin \theta \cos ^{2} \phi}\right)
\end{aligned}
$$

\section{$(2+1)$ D Relativistic spacetimes}

- Anti-de Sitter spacetime $\mathbf{A d S}^{2+1}$

$\mathcal{U}_{3}^{\mathrm{GKC}}=-\frac{k}{\tan r}+\frac{1}{\sin ^{2} r}\left(\frac{\beta_{1}}{\cosh ^{2} \theta}+\frac{\beta_{2}}{\sinh ^{2} \theta \cos ^{2} \phi}\right)$

$L_{3}=J_{01} J_{13}+J_{02} J_{23}-k \sinh \theta \sin \phi+\frac{2 \sin \phi}{\tan r}\left(\frac{\beta_{1} \sinh \theta}{\cosh ^{2} \theta}+\frac{\beta_{2}}{\sinh \theta \cos ^{2} \phi}\right)$

$\mathcal{U}_{1}^{\mathrm{GKC}}=-\frac{k}{\tan r}+\frac{1}{\sin ^{2} r \sinh ^{2} \theta}\left(\frac{\beta_{2}}{\cos ^{2} \phi}+\frac{\beta_{3}}{\sin ^{2} \phi}\right)$

$L_{1}=-J_{02} J_{12}-J_{03} J_{13}-k \cosh \theta+\frac{2 \cosh \theta}{\tan r \sinh ^{2} \theta}\left(\frac{\beta_{2}}{\cos ^{2} \phi}+\frac{\beta_{3}}{\sin ^{2} \phi}\right)$

- Minkowskian spacetime $\mathbf{M}^{2+1}$

$\mathcal{U}_{3}^{\mathrm{GKC}}=-\frac{k}{r}+\frac{1}{r^{2}}\left(\frac{\beta_{1}}{\cosh ^{2} \theta}+\frac{\beta_{2}}{\sinh ^{2} \theta \cos ^{2} \phi}\right)$

$L_{3}=J_{01} J_{13}+J_{02} J_{23}-k \sinh \theta \sin \phi+\frac{2 \sin \phi}{r}\left(\frac{\beta_{1} \sinh \theta}{\cosh ^{2} \theta}+\frac{\beta_{2}}{\sinh \theta \cos ^{2} \phi}\right)$

$\mathcal{U}_{1}^{\mathrm{GKC}}=-\frac{k}{r}+\frac{1}{r^{2} \sinh ^{2} \theta}\left(\frac{\beta_{2}}{\cos ^{2} \phi}+\frac{\beta_{3}}{\sin ^{2} \phi}\right)$

$L_{1}=-J_{02} J_{12}-J_{03} J_{13}-k \cosh \theta+\frac{2 \cosh \theta}{r \sinh ^{2} \theta}\left(\frac{\beta_{2}}{\cos ^{2} \phi}+\frac{\beta_{3}}{\sin ^{2} \phi}\right)$

- De Sitter spacetime $\mathbf{d S}^{2+1}$

$$
\begin{aligned}
& \mathcal{U}_{3}^{\mathrm{GKC}}=-\frac{k}{\tanh r}+\frac{1}{\sinh ^{2} r}\left(\frac{\beta_{1}}{\cosh ^{2} \theta}+\frac{\beta_{2}}{\sinh ^{2} \theta \cos ^{2} \phi}\right) \\
& L_{3}=J_{01} J_{13}+J_{02} J_{23}-k \sinh \theta \sin \phi+\frac{2 \sin \phi}{\tanh r}\left(\frac{\beta_{1} \sinh \theta}{\cosh ^{2} \theta}+\frac{\beta_{2}}{\sinh \theta \cos ^{2} \phi}\right) \\
& \mathcal{U}_{1}^{\mathrm{GKC}}=-\frac{k}{\tanh r}+\frac{1}{\sinh ^{2} r \sinh ^{2} \theta}\left(\frac{\beta_{2}}{\cos ^{2} \phi}+\frac{\beta_{3}}{\sin ^{2} \phi}\right) \\
& L_{1}=-J_{02} J_{12}-J_{03} J_{13}-k \cosh \theta+\frac{2 \cosh \theta}{\tanh r \sinh ^{2} \theta}\left(\frac{\beta_{2}}{\cos ^{2} \phi}+\frac{\beta_{3}}{\sin ^{2} \phi}\right)
\end{aligned}
$$

\subsubsection{Riemannian spaces}

When $\kappa_{2}=+1$ the three distances $(x, y, z)$ are completely equivalent, and their "label" in the trigonometric functions is always $\kappa_{1}$ (recall that in this case both $\theta$ and $\phi$ are ordinary 
angles). Hence the three Hamiltonians $\mathcal{H}_{i}^{\mathrm{GKC}}$ are also equivalent and we only consider a unique potential, say $\mathcal{U}_{3}^{\mathrm{GKC}}$ with constant of the motion $L_{3}$. On the spherical space $\mathbf{S}^{3}$ both $\beta_{1}, \beta_{2}$ terms can alternatively be expressed as non-central oscillators as commented in Subsection 5.1.1, meanwhile on $\mathbf{E}^{3}$ and $\mathbf{H}^{3}$ these only can be interpreted as centrifugal barriers. In this way we find the following expressions for each space:

$$
\begin{aligned}
& \mathbf{S}^{3}: \quad \mathcal{U}_{3}^{\mathrm{GKC}}=-\frac{k}{\tan r}+\frac{\beta_{1}}{\sin ^{2} x}+\frac{\beta_{2}}{\sin ^{2} y}=-\frac{k}{\tan r}+\beta_{1} \tan ^{2} r_{1}+\beta_{2} \tan ^{2} r_{2}+\beta_{1}+\beta_{2} ; \\
& \mathbf{E}^{3}: \quad \mathcal{U}_{3}^{\mathrm{GKC}}=-\frac{k}{r}+\frac{\beta_{1}}{x^{2}}+\frac{\beta_{2}}{y^{2}} ; \\
& \mathbf{H}^{3}: \quad \mathcal{U}_{3}^{\mathrm{GKC}}=-\frac{k}{\tanh r}+\frac{\beta_{1}}{\sinh ^{2} x}+\frac{\beta_{2}}{\sinh ^{2} y} .
\end{aligned}
$$

When all the $\beta_{i}=0$ we obtain the components of the Laplace-Runge-Lenz vector (6.5) for the three Riemannian spaces:

$$
\begin{aligned}
& L_{1}=-J_{02} J_{12}-J_{03} J_{13}+k \cos \theta, \\
& L_{2}=J_{01} J_{12}-J_{03} J_{23}+k \sin \theta \cos \phi, \\
& L_{3}=J_{01} J_{13}+J_{02} J_{23}+k \sin \theta \sin \phi,
\end{aligned}
$$

where the difference for each particular space comes from the translations $J_{0 i}(3.7)$ that do depend on the curvature $\kappa_{1}$.

\subsubsection{Relativistic spacetimes}

On the contrary, if $\kappa_{2}=-1$ (in units $c=1$ ), only the two space-like distances $y$ and $z$ are equivalent while $x$ is a time-like distance ( $\phi$ is also an angle for the three spacetimes but $\theta$ is a rapidity). Thus $\mathcal{U}_{2}^{\mathrm{GKC}} \simeq \mathcal{U}_{3}^{\mathrm{GKC}}$ containing a time-like centrifugal barrier and another space-like one, while $\mathcal{U}_{1}^{\mathrm{GKC}}$ defines a different potential with two space-like centrifugal barriers for the three spacetimes. By taking into account the results given in Subsection 5.1, these potentials show different superpositions of the KC potential with non-central harmonic oscillators and centrifugal barriers on $\mathbf{A} \mathbf{d} \mathbf{S}^{2+1}$ and $\mathbf{d S}^{2+1}$. The explicit expressions on each spacetime turn out to be

$$
\begin{aligned}
\mathbf{A d S}^{2+1}: \mathcal{U}_{3}^{\mathrm{GKC}} & =-\frac{k}{\tan r}+\frac{\beta_{1}}{\sin ^{2} x}+\frac{\beta_{2}}{\sinh ^{2} y}=-\frac{k}{\tan r}+\beta_{1} \tan ^{2} r_{1}+\frac{\beta_{2}}{\sinh ^{2} y}+\beta_{1}, \\
\mathcal{U}_{1}^{\mathrm{GKC}} & =-\frac{k}{\tan r}+\frac{\beta_{2}}{\sinh ^{2} y}+\frac{\beta_{3}}{\sinh ^{2} z} ; \\
\mathbf{M}^{2+1}: \quad \mathcal{U}_{3}^{\mathrm{GKC}} & =-\frac{k}{r}+\frac{\beta_{1}}{x^{2}}+\frac{\beta_{2}}{y^{2}}, \\
\mathcal{U}_{1}^{\mathrm{GKC}} & =-\frac{k}{r}+\frac{\beta_{2}}{y^{2}}+\frac{\beta_{3}}{z^{2}} ; \\
\mathbf{d S}^{2+1}: \quad \mathcal{U}_{3}^{\mathrm{GKC}} & =-\frac{k}{\tanh r}+\frac{\beta_{1}}{\sinh ^{2} x}+\frac{\beta_{2}}{\sin ^{2} y}=-\frac{k}{\tanh r}+\frac{\beta_{1}}{\sinh ^{2} x}+\beta_{2} \tan ^{2} r_{2}+\beta_{2}, \\
\mathcal{U}_{1}^{\mathrm{GKC}} & =-\frac{k}{\tanh r}+\frac{\beta_{2}}{\sin ^{2} y}+\frac{\beta_{3}}{\sin ^{2} z} \\
& =-\frac{k}{\tanh r}+\beta_{2} \tan ^{2} r_{2}+\beta_{3} \tan ^{2} r_{3}+\beta_{2}+\beta_{3} .
\end{aligned}
$$

The components of the Laplace-Runge-Lenz vector (6.5) (for $\beta_{i}=0$ ) written in terms of the kinematical generators (2.3) are

$$
\begin{aligned}
& L_{1}=-P_{1} K_{1}-P_{2} K_{2}-k \cosh \theta, \quad L_{2}=P_{0} K_{1}-P_{2} J-k \sinh \theta \cos \phi, \\
& L_{3}=P_{0} K_{2}+P_{1} J-k \sinh \theta \sin \phi .
\end{aligned}
$$




\section{Concluding remarks}

We have achieved the generalization of the 3D Euclidean superintegrable family (1.1) as well as the maximally superintegrable SW (1.2) and GKC (1.3) potentials to the space $\mathbb{S}_{\left[\kappa_{1}\right] \kappa_{2}}^{3}$ by applying a unified approach which makes use of a built-in scheme of contractions. Furthermore the results so obtained have been described and interpreted on each particular space and have also been displayed along the paper in tabular form. Thus we have explicitly shown that (maximal) superintegrability is preserved for any value of the curvature and for either a Riemannian or Lorentzian metric. Notice that on the complex sphere (see e.g. [31]) and on the ambient space $\mathbb{R}^{4}$ these two maximally superintegrable Hamiltonians read

$$
\mathcal{H}^{\mathrm{SW}}=\sum_{\mu=0}^{3}\left(\frac{1}{2} p_{\mu}^{2}+\frac{\beta_{\mu}}{x_{\mu}^{2}}\right)-\beta_{0}, \quad \mathcal{H}_{3}^{\mathrm{GKC}}=\frac{1}{2} \sum_{\mu=0}^{3} p_{\mu}^{2}-\frac{k x_{0}}{\sqrt{x_{1}^{2}+x_{2}^{2}+x_{3}^{2}}}+\frac{\beta_{1}}{x_{1}^{2}}+\frac{\beta_{2}}{x_{2}^{2}},
$$

where $\sum_{\mu=0}^{3} x_{\mu}^{2}=1$. Therefore the Hamiltonians here studied can be regarded as different real forms coming from known complex superintegrable systems through graded contractions, that is, by introducing the parameters $\kappa_{1}$ and $\kappa_{2}$.

As far as the superintegrable potential $\mathcal{U}(4.1)$ is concerned, we recall that in this $3 \mathrm{D}$ case, we have one constant of the motion (besides de Hamiltonian) more than the two ones that ensure its complete integrability, but one integral less than the four ones that determine maximal superintegrability. By taking into account the former point of view one may claim that $\mathcal{U}$ is minimally (or weak) superintegrable, while from the latter, $\mathcal{U}$ would be quasi-maximally superintegrable. Our opinion is that when the corresponding Hamiltonian $\mathcal{H}=\mathcal{T}+\mathcal{U}$ is constructed on the $N D$ spaces $\mathbb{S}_{\left[\kappa_{1}\right] \kappa_{2}}^{N}$, each of the $N(N-1)$ generators $J_{i j}(i, j=1, \ldots, N ; i<j)$ of the (Lorentz) rotation subalgebra $s o_{\kappa_{2}}(N)$ would provide a constant of the motion $I_{i j}$ of the type (4.2). Next, by following [9, 23], two subsets of $N-1$ constants of the motion, $Q_{(l)}$ and $Q^{(l)}$, should be deduced from the initial set of $N(N-1)$ integrals as:

$$
Q^{(l)}=\sum_{i, j=1}^{l} I_{i j}, \quad Q_{(l)}=\sum_{i, j=N-l+1}^{N} I_{i j}, \quad l=2, \ldots, N
$$

where $Q^{(N)} \equiv Q_{(N)}$. In this way the complete integrability of $\mathcal{H}$ would be characterized by either the $N$ constants of the motion $\left\{Q^{(l)}, \mathcal{H}\right\}$ or by $\left\{Q_{(l)}, \mathcal{H}\right\}$. The quasi-maximal superintegrability would be provided by the $2 N-2$ functions

$$
\left\{Q^{(2)}, Q^{(3)}, \ldots, Q^{(N)} \equiv Q_{(N)}, \ldots, Q_{(3)}, Q_{(2)}, \mathcal{H}\right\}
$$

The corresponding SW potential on $\mathbb{S}_{\left[\kappa_{1}\right] \kappa_{2}}^{N}$ would be obtained by taking the same $\mathcal{F}(r)$ as in (5.1) and the remaining constant of the motion would come from one of the translation generators $J_{0 i}$ in the form $I_{0 i}$ (5.3). Likewise, a set of $N$ GKC potentials could be constructed by starting from the radial function (6.1) and then taking $N-1$ centrifugal terms for each of them as in (6.2); in this case the additional constant of the motion $L_{i}$ would be of the form (6.3).

We stress that this scheme of the possible $N D$ generalization of all the $3 \mathrm{D}$ results here presented (currently in progress) relies on the fact that the potential $\mathcal{U}$ can be endowed with a coalgebra symmetry [10]. This indeed allowed us to obtain the integrals (7.7) for the ND SW system on the three Riemannian spaces in $[9,23]$ by starting from the quantum deformation of the Euclidean SW system introduced in [1,2]. Furthermore, quantum deformations have been shown $[6,7]$ to give rise to Riemannian and relativistic spaces of non-constant curvature on which SW- and KC-type potentials can be considered [8]. 


\section{Acknowledgements}

This work was partially supported by the Ministerio de Educación y Ciencia (Spain, Project FIS2004-07913) and by the Junta de Castilla y León (Spain, Projects BU04/03 and VA013C05).

[1] Ballesteros A., Herranz F.J., Integrable deformations of oscillator chains from quantum algebras, J. Phys. A: Math. Gen., 1999, V.32, N 50, 8851-8862; solv-int/9911004.

[2] Ballesteros A., Herranz F.J., Musso F., Ragnisco O., Superintegrable deformations of the SmorodinskyWinternitz Hamiltonian, in Superintegrability in Classical and Quantum Systems, Editors P. Tempesta, P. Winternitz, J. Harnad, W. Miller Jr., G. Pogosyan and M.A. Rodríguez, CRM Proceedings and Lecture Notes, Providence, American Mathematical Society, 2004, V.37, 1-14; math-ph/0412067.

[3] Ballesteros A., Herranz F.J., del Olmo M.A., Santander M., Quantum structure of the motion groups of the two-dimensional Cayley-Klein geometries, J. Phys. A: Math. Gen., 1993, V.26, N 21, 5801-5823.

[4] Ballesteros A., Herranz F.J., del Olmo M.A., Santander M., Quantum (2+1) kinematical algebras: a global approach, J. Phys. A: Math. Gen., 1994, V.27, N 4, 1283-1297.

[5] Ballesteros A., Herranz F.J., del Olmo M.A., Santander M., Classical deformations, Poisson-Lie contractions, and quantization of dual Lie bialgebras, J. Math. Phys., 1995, V.36, N 2, 631-640.

[6] Ballesteros A., Herranz F.J., Ragnisco O., Curvature from quantum deformations, Phys. Lett. B, 2005, V.610, N 1-2, 107-114; hep-th/0504065.

[7] Ballesteros A., Herranz F.J., Ragnisco O., Integrable geodesic motion on 3D curved spaces from non-standard quantum deformations, Czech. J. Phys., 2005, V.55, N 11, 1327-1333; math-ph/0508038.

[8] Ballesteros A., Herranz F.J., Ragnisco O., Integrable potentials on spaces with curvature from quantum groups, J. Phys. A: Math. Gen., 2005, V.38, N 32, 7129-7144; math-ph/0505081.

[9] Ballesteros A., Herranz F.J., Santander M., Sanz-Gil T., Maximal superintegrability on $N$-dimensional curved spaces, J. Phys. A: Math. Gen., 2003, V.36, N 7, L93-L99; math-ph/0211012.

[10] Ballesteros A., Ragnisco O., A systematic construction of integrable Hamiltonians from coalgebras, J. Phys. A: Math. Gen., 1998, V.31, N 16, 3791-3813; solv-int/9802008.

[11] Cariñena J.F., Rañada M.F., Santander M., Central potentials on spaces of constant curvature: The Kepler problem on the two-dimensional sphere $S^{2}$ and the hyperbolic plane $H^{2}$, J. Math. Phys., 2005, V.46, N 5, 052702, 18 pages; math-ph/0504016.

[12] Cariñena J.F., Rañada M.F., Santander M., Sanz-Gil T., Separable potentials and a triality in twodimensionl spaces of constant curvature, J. Nonlinear Math. Phys., 2005, V.12, N 2, 230-252.

[13] Doubrovine B., Novikov S., Fomenko A., Géométrie Contemporaine, Méthodes et Applications, Part 1, Traduit du Russe, Mathematiques, Moscow, Mir, 1985 (in French).

[14] Evans N.W., Superintegrability in classical mechanics, Phys. Rev. A, 1990, V.41, N 10, 5666-5676.

[15] Evans N.W., Superintegrability of the Winternitz system, Phys. Lett. A, 1990, V.147, N 8-9, 483-486.

[16] Evans N.W., Group theory of the Smorodinsky-Winternitz system, J. Math. Phys., 1991, V.32, N 12, 3369-3375.

[17] Fris J., Mandrosov V., Smorodinsky Y.A., Uhlir M., Winternitz P., On higher symmetries in quantum mechanics, Phys. Lett., 1965, V.16, N 3, 354-356.

[18] Gromov N.A., Man'ko V.I., The Jordan-Schwinger representations of Cayley-Klein groups. I. The orthogonal groups, J. Math. Phys., 1990, V.31, N 5, 1047-1053.

[19] Grosche C., Pogosyan G.S., Sissakian A.N., Path integral discussion for Smorodinsky-Winternitz potentials 1. Two- and three-dimensional Euclidean space, Fortschr. Phys., 1995, V.43, N 6, 453-521; hep-th/9402121.

[20] Grosche C., Pogosyan G.S., Sissakian A.N., Path integral discussion for Smorodinsky-Winternitz potentials 2. The two- and three-dimensional sphere, Fortschr. Phys., 1995, V.43, N 6, 523-563.

[21] Grosche C., Pogosyan G.S., Sissakian A.N., Path integral approach for superintegrable potentials on the three-dimensional hyperboloid, Phys. Part. Nuclei, 1997, V.28, N 5, 486-519.

[22] Helgason S., Differential geometry and symmetric spaces, New York, Academic Press, 1962.

[23] Herranz F.J., Ballesteros A., Santander M., Sanz-Gil T., Maximally superintegrable Smorodinsky-Winternitz systems on the $N$-dimensional sphere and hyperbolic spaces, in Superintegrability in Classical and Quantum Systems, Editors P. Tempesta, P. Winternitz, J. Harnad, W. Miller Jr., G. Pogosyan and M.A. Rodríguez, CRM Proceedings and Lecture Notes, Providence, American Mathematical Society, 2004, V.37, 75-89; math-ph/0501035. 
[24] Herranz F.J., Ortega R., Santander M., Trigonometry of spacetimes: a new self-dual approach to a curvature/signature (in)dependent trigonometry, J. Phys. A: Math. Gen., 2000, V.33, N 24, 4525-4551; for an extended version see math-ph/9910041.

[25] Herranz F.J., Santander M., Conformal symmetries of spacetimes, J. Phys. A: Math. Gen., 2002, V.35, N 31, 6601-6618; math-ph/0110019.

[26] Higgs P.W., Dynamical symmetries in a spherical geometry I, J. Phys. A: Math. Gen., 1979, V.12, N 3, 309-323.

[27] Izmest'ev A.A., Pogosyan G.S., Sissakian A.N., Winternitz P., Contractions of Lie algebras and separation of variables. The $n$-dimensional sphere, J. Math. Phys., 1999, V.40, N 3, 1549-1573.

[28] Kalnins E.G., Kress J.M.,, Pogosyan G.S., Miller W., Completeness of superintegrability in two-dimensional constant-curvature spaces, J. Phys. A: Math. Gen., 2001, V.34, N 22, 4705-4720; math-ph/0102006.

[29] Kalnins E.G., Miller W., Hakobyan Y.M., Pogosyan G.S., Superintegrability on the two-dimensional hyperboloid II, J. Math. Phys., 1999, V.40, N 5, 2291-2306; quant-ph/9907037.

[30] Kalnins E.G., Miller W., Pogosyan G.S., Superintegrability of the two-dimensional hyperboloid, J. Math. Phys., 1997, V.38, N 10, 5416-5433.

[31] Kalnins E.G., Miller W., Pogosyan G.S., Completeness of multiseparable superintegrability on the complex 2-sphere, J. Phys. A: Math. Gen., 2000, V.33, N 38, 6791-6806.

[32] Kalnins E.G., Miller W., Pogosyan G.S., Coulomb-oscillator duality in spaces of constant curvature, J. Math. Phys., 2000, V.41, N 5, 2629-2657; quant-ph/9906055.

[33] Kalnins E.G., Miller W., Pogosyan G.S., The Coulomb-oscillator relation on $n$-dimensional spheres and hyperboloids, Phys. Atomic Nuclei, 2002, V.65, N 6, 1086-1094; math-ph/0210002.

[34] Kalnins E.G., Pogosyan G.S., Miller W., Completeness of multiseparable superintegrability in two dimensions, Phys. Atomic Nuclei, 2002, V.65, N 6, 1033-1035.

[35] Kalnins E.G., Williams G.C., Miller W., Pogosyan G.S., Superintegrability in three-dimensional Euclidean space, J. Math. Phys., 1999, V.40, N 2, 708-725.

[36] Leemon H.I., Dynamical symmetries in a spherical geometry II, J. Phys. A: Math. Gen., 1979, V.12, N 4, 489-501.

[37] Nersessian A., Pogosyan G., Relation of the oscillator and Coulomb systems on spheres and pseudospheres, Phys. Rev. A, 2001, V.63, N 2, 020103; quant-ph/0006118.

[38] Perelomov A.M., Integrable systems of classical mechanics and Lie algebras, Berlin, Birkhäuser, 1990.

[39] Rañada M.F., Santander M., Superintegrable systems on the two-dimensional sphere $S^{2}$ and the hyperbolic plane $H^{2}$, J. Math. Phys., 1999, V.40, N 10, 5026-5057.

[40] Rañada M.F., Santander M., On some properties of harmonic oscillator on spaces of constant curvature, Rep. Math. Phys., 2002, V.49, N 2-3, 335-343.

[41] Rañada M.F., Santander M., On harmonic oscillators on the two-dimensional sphere $S^{2}$ and the hyperbolic plane $H^{2}$, J. Math. Phys., 2002, V.43, N 1, 431-451.

[42] Rañada M. F., Santander M., On harmonic oscillators on the two-dimensional sphere $S^{2}$ and the hyperbolic plane $H^{2}$ II, J. Math. Phys., 2003, V.44, N 5, 2149-2167.

[43] Schrödinger E., A method of determining quantum mechanical eigenvalues and eigenfunctions, Proc. R. Ir. Acad. A, 1940, V.46, 9-16. 